\title{
Minimal-Variance Hedging in Large Financial Markets: random fields approach
}

\author{
Giulia Di Nunno* and Inga Baadshaug Eide* \\ Revised in December 2008
}

\begin{abstract}
We study a large financial market where the discounted asset prices are modeled by martingale random fields. This approach allows the treatment of both the cases of a market with a countable amount of assets and of a market with a continuum amount. We discuss conditions for these markets to be complete and we study the minimal variance hedging problem both in the case of full and partial information. An explicit representation of the minimal variance hedging portfolio is suggested. Techniques of stochastic differentiation are applied to achieve the main results. Examples of large market models with a countable number of assets are considered according to the literature and an example of market model with a continuum of assets is taken from the bond market.
\end{abstract}

AMS subject classification: 60H05, 60G57, 60G60, 91B24, 91B28

Key-words: large market, bond market, minimal variance hedging, random field, martingale random field, stochastic derivative.

\section{Introduction}

Large financial markets were first introduced by Kabanov and Kramkov in [23] as a sequence of finite dimensional markets, called "small markets" in [31]. Each small market is defined on its own probability space, filtration and time horizon. With this approach the large financial market can be seen as a market where it is possible to choose a finite number of securities to trade, but this number is not a priori bounded. In this framework asymptotic arbitrage and the corresponding versions of the fundamental theorem

\footnotetext{
${ }^{*}$ Centre of Mathematics for Applications (CMA), Department of Mathematics, University of Oslo, P.O. Box 1053 Blindern, N-0316 Oslo Norway. Email: giulian@math.uio.no, ingae@math.uio.no
} 
of asset pricing have been studied. The pioneering papers [23] and [24] provide a first extension of this fundamental theorem connecting the concepts of asymptotic arbitrage with the notion of contiguity of a sequence of equivalent martingale measures. A general version of this theorem is then given in [29], where the concept of asymptotic free lunch is introduced - see also [30], [31]. The relationship between "no-arbitrage" and "economic equilibrium" in a market with a countable number of assets is found e.g. in [32] and also $[2],[22]$.

If we assume that all the probability spaces where the small markets are defined coincide, then we have an alternative approach. One can define a large market as a countable number of assets and, correspondingly, a sequence of price processes on one fixed probability space, filtration and time horizon. This is a model for an idealized market in which it is allowed to trade on countably many assets. This framework is more suitable for considering questions related to completeness and hedging problems. In fact it has been chosen in e.g. [5] and [8] where questions related the completion of the market and portfolio diversification are considered and in e.g. [10] and [7] where utility maximization and mean-variance hedging are considered.

A claim on a future wealth in a financial market is said to be attainable if it can be perfectly replicated by self-financed trading in assets available on the market. A financial market is said to be complete if any possible claim is attainable. An example of a complete market is the classical BlackScholes market. In general, however, markets are not complete and there are claims for which there is no perfectly replicating trading strategy. In this case one can try to find trading strategies whose final payoff is in some sense the closest to the initial claim. We need to make these concepts precise.

We consider a risk neutral market model on the complete probability space $(\Omega, \mathcal{F}, P)$ equipped with a filtration $\mathbb{F}:=\left\{\mathcal{F}_{t}, t \geq 0\right\}$ representing the flow of information associated to the market events. Let $T>0$ be a fixed time horizon and the discount factor be identically equal to 1 . Then a claim $\xi$ is a random variable such that its discounted value is square integrable with respect to the risk neutral measure $P$. Moreover, the claim $\xi$ is attainable if it is the payoff at $T$ of a self-financing portfolio $\phi$ for a given initial endowment $w$ and such that the associated discounted value process is a martingale with respect to $\mathbb{F}$ under $P$ - see e.g. [26]. Note that $w$ equals the expected discounted payoff. Denote by $\mathcal{H}$ the set of claims that are attainable on the market. A natural candidate for a best approximation to the claim $\xi$ is the solution $\widehat{\xi}$ to

$$
\min _{\theta \in \mathcal{H}} E\left[(\xi-\theta)^{2}\right]=\min _{\substack{\theta \in \mathcal{H} \\ E[\theta]=E[\xi]}} \operatorname{var}(\xi-\theta) .
$$


The problem of finding $\widehat{\xi}$ and the corresponding self-financing trading strategy is commonly referred to as the problem of minimal variance hedging (cf. e.g. [1]) or mean-variance hedging (cf. e.g. [43]). The trading strategy generating the solution to (1.1) is referred to as a minimal variance (or mean-variance) hedging strategy for $\xi$ or also the variance optimal trading strategy for $\xi([20])$. The problem (1.1) has been extensively studied and we refer to [1], [14] and [43] for overviews. The results rely heavily on the representation of payoffs as stochastic integrals and naturally the choice of framework of stochastic integration is crucial. Accordingly, the extensions to large financial markets have to be carried through carefully.

An example of a large market with a continuum of assets is a bond market with an infinity of different maturities. Such markets are studied in [4], [9] and [11]. In [4] the authors suggest two different ways to construct a stochastic integral with respect to price processes taking values in the space of continuous processes: one based on the concept of controlled processes as integrators and another one "tailor made" for jump-diffusion price processes. Apart from some differences in hypotheses, the resulting integrals are the same. One admissible strategy, in this setting, is the permanent reinvestment of the whole portfolio value in the bond that is about to mature. This strategy gives the same return as the short rate of interest, which makes the assumption of a "bank account" paying the short rate of interest superfluous. Some Heath-Jarrow-Morton type of conditions are established for the existence of an equivalent martingale measure. The authors also consider market completeness and introduce the notion of "approximate completeness" which is then proved to be equivalent to uniqueness of the equivalent martingale measure. In [11] the framework of cylindrical stochastic integration is taken. However the authors conclude that the space strategies may be insufficient when discussing problems of hedging and completeness in view of the fact that the space of measure-valued integrands is incomplete. The authors also give an analysis of Kennedy's model of forward rates in terms of a Gaussian random field (cf. [27],[28]). This idea is taken further in [9].

In this paper we study a large market model that is risk neutral in the sense that the discounted asset prices are martingales with respect to the physical measure. For this market model we consider the minimal variance hedging problem. In [7], the problem (1.1) is studied in a market with a countable number of assets, using the method suggested in [21] and the construction for stochastic integrals with respect to a sequence of semimartingales as given in [12] (see also cylindrical integration in [36]). Our method is substantially different both for choice of integration framework (we use an Itô type integral with respect to a martingale random field) and characterization of the hedging strategy (given in terms of non-anticipating stochastic derivative). 
In order to deal with an infinite number of assets, we consider each asset to be indexed by $x$ and we denote $\mathbb{X}$ the set of indexes. We interpret the set $\mathbb{X}$ as a topological space. Thus in a small financial market, i.e. a market with a finite number $N$ of assets available, we have $\mathbb{X}=\{1, \ldots, N\}$ and in a large market with a countable number of assets available we have $\mathbb{X}=\{1,2, \ldots\}$. Both these cases correspond to discrete topological spaces. In the general setting, if $x \in \mathbb{X}$ denotes the single security, a set $B \in \mathfrak{B}_{\mathbb{X}}$ is interpreted as a group of securities which will be called package $B$. In the risk neutral framework, the discounted prices are given by a martingale random field $\mu=\mu(x, t),(x, t) \in \mathbb{X} \times[0, T]$, which is at the base of our model.

The paper is organized as follows. In Section 2 we introduce the concept of martingale random field and examples are provided. Section 3 is dedicated to non-anticipating integration and differentiation. In particular we study the non-anticipating derivative in the framework suggested. This generalizes [14] and [13]. The non-anticipating stochastic derivative is naturally linked to the minimal variance hedging strategy as it is shown in the following section. Section 4 is dedicated to the market model and questions related to completeness of the market and minimal variance hedging are studied. The optimization problem is formulated both with respect to final payoffs to be achieved at the time horizon $T$ and for processes of payoffs to be achieved within the time interval $[0, T]$. The use of the non-anticipating derivative allows an explicit characterization of the minimal variance hedging strategy. Throughout the paper we deal with a reference filtration $\mathbb{F}=\left\{\mathcal{F}_{t}, 0 \leq t \leq\right.$ $T$ \} representing the information available on the market and the one the agents can rely on in their decision making process. If a trader has less information available, we say that he is in a situation of partial information. In this case he relies on the flow of information $\mathbb{E}=\left\{\mathcal{E}_{t}, 0 \leq t \leq T\right\}$ with $\mathcal{E}_{t} \subseteq \mathcal{F}_{t}$. A characterization of minimal variance hedging in the case of partial information setting is also given by means of the non-anticipating derivative. The final Section 5 presents two examples of large market: one with a countable number of assets available (also treated e.g. in [23] and [5]), and one taken by the bond market with a continuum of assets.

\section{Martingale random fields}

Consider a fixed time horizon $T>0$ and a complete probability space $(\Omega, \mathcal{F}, P)$ equipped with the right-continuous filtration

$$
\mathbb{F}:=\left\{\mathcal{F}_{t}, 0 \leq t \leq T\right\}
$$

with $\mathcal{F}_{t}$ representing the information available on the market at time $t$. We assume $\mathcal{F}_{0}$ to be trivial up to $P$-null events and, for simplicity in notation, we set $\mathcal{F}=\mathcal{F}_{T}$. Let $L_{2}(P):=L_{2}(\Omega, \mathcal{F}, P)$ be the standard space of random 
variables with finite norm

$$
\|\xi\|_{L_{2}(P)}:=\left(E\left[\xi^{2}\right]\right)^{1 / 2} .
$$

Let $\mathbb{X}$ be a topological space equipped with the separable (i.e. countably generated) Borel $\sigma$-algebra $\mathfrak{B}_{\mathbb{X}}$. We assume $\mathfrak{B}_{\mathbb{X}}$ to be generated by the countable semi-ring $\mathfrak{B}$ (see e.g. [3, p. 166]) of sets in $\mathbb{X}$. The Borel $\sigma$-algebra on $(0, T]$ (also separable) is denoted $\mathfrak{B}_{(0, T]}$. Note that this $\sigma$-algebra can be regarded as generated by a the semi-ring of intervals of the form $(s, u]$ where $0 \leq s<u \leq T$. Thus the sets

$$
\Delta=B \times(s, u], \quad B \in \mathfrak{B}, 0 \leq s<u \leq T,
$$

generate $\mathfrak{B}_{\mathbb{X}} \times \mathfrak{B}_{(0, T]}$.

We denote $\mathfrak{P}$ the predictable $\sigma$-algebra on $\Omega \times \mathbb{X} \times[0, T]$, i.e. the $\sigma$-algebra generated by sets of the form

$$
F \times B \times(s, u], \quad F \in \mathcal{F}_{s}, B \in \mathfrak{B}, 0 \leq s<u \leq T .
$$

We introduce the following definition.

Definition 2.1. A stochastic set-function

$$
\mu(\Delta), \quad \Delta \in \mathfrak{B}_{\mathbb{X}} \times \mathfrak{B}_{[0, T]},
$$

is a martingale random field (with orthogonal values) with respect to $\mathbb{F}$ if it satisfies the following properties:

(i) there exists some tight ${ }^{1} \sigma$-finite measure $m$ on $\mathfrak{B}_{\mathbb{X}} \times \mathfrak{B}_{[0, T]}$ such that

$$
m(\Delta)=E\left[\mu^{2}(\Delta)\right], \quad \Delta \in \mathfrak{B}_{\mathbb{X}} \times \mathfrak{B}_{[0, T]},
$$

and $m(\mathbb{X} \times\{0\})=0$. The measure $m$ is hereafter called the variance measure.

(ii) it is additive, i.e. for any pairwise disjoint $\Delta_{1}, \ldots, \Delta_{K} \in \mathfrak{B}_{\mathbb{X}} \times \mathfrak{B}_{[0, T]}$,

$$
\mu\left(\bigsqcup_{k=1}^{K} \Delta_{k}\right)=\sum_{k=1}^{K} \mu\left(\Delta_{k}\right)
$$

(iii) it is $\mathbb{F}$-adapted, i.e. for any $t$ and for any $\Delta \in \mathfrak{B}_{\mathbb{X}} \times \mathfrak{B}_{[0, t]}$, the value $\mu(\Delta)$ is an $\mathcal{F}_{t}$-measurable random variable,

\footnotetext{
${ }^{1}$ Recall that a measure is tight if for every $\delta>0$ there exists a compact $X_{\delta}$ such that $m\left(X \backslash X_{\delta}\right)<\delta$.
} 
(iv) it has the martingale property, i.e. for any $0 \leq t \leq T$ and any $\Delta \in$ $\mathfrak{B}_{\mathbb{X}} \times \mathfrak{B}_{(t, T]}$ such that $m(\Delta)<\infty$ we have

$$
E\left[\mu(\Delta) \mid \mathcal{F}_{t}\right]=0 .
$$

Moreover, we assume that the martingale random field

(v) has conditionally orthogonal values, i.e. if $\Delta_{1}, \Delta_{2} \in \mathfrak{B}_{\mathbb{X}} \times \mathfrak{B}_{(t, T]}$ are disjoint and $m\left(\Delta_{1}\right), m\left(\Delta_{2}\right)<\infty$, then

$$
E\left[\mu\left(\Delta_{1}\right) \mu\left(\Delta_{2}\right) \mid \mathcal{F}_{t}\right]=0 .
$$

Note that (i) and (iii) yield $\mu(\mathbb{X} \times\{0\})=0$. Note also that we use the term set-function and not stochastic measure because we do not assume that for every $\omega \in \Omega$ the function $\mu(\omega, \Delta), \Delta \in \mathfrak{B}_{\mathbb{X}} \times \mathfrak{B}_{[0, T]}$, is a measure.

Remark 2.1. The martingale random field is $\sigma$-additive in $L_{2}(P)$ in the sense that for any pairwise disjoint sets $\Delta_{1}, \Delta_{2}, \ldots$ in $\mathfrak{B}_{\mathbb{X}} \times \mathfrak{B}_{[0, T]}$ such that $m\left(\bigsqcup_{k} \Delta_{k}\right)<\infty$, we have

$$
\mu\left(\bigsqcup_{k=1}^{\infty} \Delta_{k}\right)=\sum_{k=1}^{\infty} \mu\left(\Delta_{k}\right)
$$

with convergence in $L_{2}(P)$. The $\sigma$-finiteness of $m$ implies that there is some collection of sets $A_{n}, n=1,2, \ldots$, such that $\mathbb{X} \times[0, T]=\bigcup_{n} A_{n}$ and $E\left[\mu^{2}\left(A_{n}\right)\right]<\infty$. Being this property a form of $\sigma$-finiteness for the setfunction $\mu$, we call it $\sigma$-finiteness in $L_{2}(P)$.

Remark 2.2. The $\sigma$-finiteness of $m$ ensures that the (separable) $\sigma$-algebra $\mathfrak{B}_{\mathbb{X}} \times \mathfrak{B}_{[0, T]}$ can be (countably) generated by the semi-ring sets $\Delta$ of type (2.2) having $m(\Delta)<\infty$. We will exploit these facts in the proof of Theorem 2.1 .

Remark 2.3. Martingale (or martingale-difference) random fields were first introduced in mathematical statistics as forms of multi-parameter martingales already in the 70's and developed further in the 80's. Most of the studies concern ergodic properties and limit theorems. Various different definitions can be found in the literature according to the different interpretations of how the flow of information $\mathbb{F}$ and the type of ordering in the index set is taken into account within the "martingale property". In our case the ordering is the natural one given by "time". In the frame of stochastic calculus with respect to martingale random fields we have to recall the work by Wong and Zakai [44], Cairoli and Walsh [6]. In their terminology our martingale random field is both a "strong" and a "weak" martingale. In fact we only have one natural reference filtration. Their work aimed to develop a non-anticipating calculus for different martingale-difference random fields on $R^{2}$. However, most of the developments are achieved in the case of the so-called Brownian sheet. Besides, other form of calculus are developed, e.g. line integrals. 
Theorem 2.1. Suppose that $\mu$ is a martingale random field with orthogonal values and define the set-function

$$
P M(F \times \Delta)=E\left[\chi_{F} \mu^{2}(\Delta)\right]
$$

on sets of type $F \times \Delta=F \times B \times(s, u]$ in $\mathfrak{P}$. Then $P M$ extends to a measure on $\mathfrak{P}$ which admits representation in the product form

$$
P M(d \omega d x d t)=P(d \omega) \times M(\omega, d x d t)
$$

where $M(\omega, \cdot)$ is a $\sigma$-finite measure on $\mathfrak{B}_{\mathbb{X}} \times \mathfrak{B}_{[0, T]}$, depending on $\omega$ as a parameter. The stochastic measure $M$ is unique in the sense that any other stochastic measure satisfying (2.7)-(2.8) would have P-a.s. the same trajectories as $M$. The stochastic measure $M$ is hereafter called the conditional variance measure of $\mu$.

Proof. Recall that the sets of type (2.3) constitute a semi-ring generating the $\sigma$-algebra $\mathfrak{P}$. The set-function $P M(2.7)$ is non-negative, additive and $\sigma$-finite on the sets (2.3), i.e. for $\Gamma_{k}:=F_{k} \times \Delta_{k}, k=1,2, \ldots$, pairwise disjoint sets of type (2.3) such that $\Gamma:=\bigsqcup_{k} \Gamma_{k}$ is also of type (2.3), with representation $\Gamma=F \times \Delta$, we have $P M(\Gamma)=\sum_{k} P M\left(\Gamma_{k}\right)$. In fact, by Remark 2.1

$$
\begin{aligned}
P M(\Gamma) & =E\left[1_{F} \mu^{2}(\Delta)\right] \\
& =E\left[\left(\sum_{k} \int_{\mathbb{X} \times[0, T]} \chi_{F_{k}} \chi_{\Delta_{k}}(x, t) \mu(d x d t)\right)^{2}\right] \\
& =\sum_{k} E\left[\left(\chi_{F_{k}} \mu\left(\Delta_{k}\right)\right)^{2}\right] \\
& =\sum_{k} P M\left(\Gamma_{k}\right) .
\end{aligned}
$$

Note that for any $\Gamma=F \times \Delta$ of type (2.3) such that $m(\Delta)<\infty$ we have that $P M(\Gamma)<\infty$. Moreover, since $m$ is $\sigma$-finite, then also $P M(2.7)$ is $\sigma$-finite on the sets of the semi-ring (2.3). Thus $P M$ extends (uniquely) to a $\sigma$-finite measure on the $\sigma$-algebra $\mathfrak{P}$ that is generated by the sets $(2.3)$. See [3, Theorem 11.3, Theorem 10.3].

Let us consider a semi-ring set $\Delta=B \times(s, u]$ of type (2.2) with $m(\Delta)<\infty$, see Remark 2.2. The process given by

$$
\mu_{t}(\Delta):=\mu(B \times(s, t]), \quad t \in(s, u],
$$

and $\mu_{s}(\Delta):=\mu(B \times\{s\})$ is a square integrable martingale. Let

$$
M_{t}(\Delta):=<\mu \cdot(\Delta)>_{t}, \quad t \in(s, u],
$$


denote the (unique) predictable stochastic process with non-decreasing trajectories, $M_{s}(\Delta)=0$, such that

$$
\mu_{t}^{2}(\Delta)-M_{t}(\Delta), \quad 0 \leq t \leq T,
$$

is a martingale. See Doob-Meyer decomposition theorem as in e.g. [41, VI 34 , p. 376]. The process $M_{t}(\Delta), t \in[s, u]$, is unique in the sense that if there exists another process $\widetilde{M}_{t}(\Delta), t \in[s, u]$, with the same properties, then $P\left\{M_{t}(\Delta)=\widetilde{M}_{t}(\Delta)\right.$, for all $\left.t \in[s, u]\right\}=1$. Moreover, for any $F \in \mathcal{F}_{s}$, we have

$$
\begin{aligned}
P M(F \times \Delta) & =E\left[\chi_{F} \mu^{2}(\Delta)\right] \\
& =E\left[\chi_{F} \mu_{u}^{2}(\Delta)\right] \\
& =E\left[\chi_{F} M_{u}(\Delta)\right] .
\end{aligned}
$$

From the semi-ring properties and the additivity of $\mu$ we see that

$$
E\left[\chi_{F} M_{u}(\Delta)\right]=E\left[\chi_{F} \sum_{k=1}^{K} M_{u_{k}}\left(\Delta_{k}\right)\right], \quad F \in \mathcal{F}_{s},
$$

for $\Delta=\bigsqcup_{k=1}^{K} \Delta_{k}$ with $\Delta_{k}=B_{k} \times\left(s_{k}, u_{k}\right]$. From (2.11) we define the non-negative set-function

$$
M(\Delta):=M_{u}(\Delta), \quad \Delta=B \times(s, u],
$$

on the semi-ring sets $(2.2)$ with $m(B \times(s, u])<\infty$, see Remark 2.2. Naturally,

$$
P M(F \times \Delta)=E\left[\chi_{F} M(B \times(s, u])\right] .
$$

From (2.12) we see that the set-function $M$ is additive and $\sigma$-additive in $L_{1}(P)$ on the sets $(2.2)$ in the sense that, for any $\Delta_{k}=B_{k} \times\left(s_{k}, u_{k}\right], k=$ $1,2, \ldots$, pairwise disjoint elements with $m\left(\Delta_{k}\right)<\infty$ such that $\Delta:=\bigsqcup_{k} \Delta_{k}$ is also of type (2.2), i.e. $\Delta=B \times(s, u]$ where $s=\inf _{k} s_{k}$, then for any $F \in \mathcal{F}_{s}$ we have that

$$
\begin{aligned}
E\left[\chi_{F} M(\Delta)\right] & =P M(F \times \Delta) \\
& =\sum_{k} P M\left(F \times \Delta_{k}\right) \\
& =E\left[\chi_{F} \sum_{k} M\left(\Delta_{k}\right)\right],
\end{aligned}
$$

thus $M(\Delta)=\sum_{k} M\left(\Delta_{k}\right)$ on $\mathcal{F}_{s}$ with convergence in $L_{1}(P)$. We can set

$$
M(B \times\{0\})=0, \quad B \in \mathfrak{B} .
$$


Note that since $m$ is $\sigma$-finite and $\mathfrak{B}_{\mathbb{X}} \times \mathfrak{B}_{[0, T]}$ is generated by the semi-ring sets having finite measure $m$ (see Remark 2.2), the set-function $M$ is also $\sigma$-finite in $L_{1}(P)$ on the sets (2.2), i.e. there exist $\Delta_{n}, n=1,2, \ldots$, of type (2.2) such that $\mathbb{X} \times[0, T]=\bigcup_{n} \Delta_{n}$ and $E\left[M\left(\Delta_{n}\right)\right]<\infty$ (cf. Remark 2.1). Hence the set-function $M$ in (2.13) extends to a non-negative, $\sigma$-additive, $\sigma$-finite (in $L_{1}(P)$ ) set-function on the $\sigma$-algebra $\mathfrak{B}_{\mathbb{X}} \times \mathfrak{B}_{(0, T]}$ generated by (2.2). The values

$$
M(\Delta), \quad \Delta \in \mathfrak{B}_{\mathbb{X}} \times \mathfrak{B}_{(0, T]},
$$

are random variables and, if $m(\Delta)<\infty$, then $E[M(\Delta)]<\infty$.

Now it only remains to show that the set-function (2.15) admits a regular modification $\widetilde{M}$ so that, for any $\omega \in \Omega$,

$$
\widetilde{M}(\omega, \Delta), \quad \Delta \in \mathfrak{B}_{\mathbb{X}} \times \mathfrak{B}_{[0, T]},
$$

is a measure and then

$$
P M(\Gamma)=\iint_{\Gamma} \widetilde{M}(\omega, d x d t) P(d \omega), \quad \Gamma \in \mathfrak{P},
$$

and in particular

$$
P M(F \times \Delta)=\int_{F} M(\omega, \Delta) P(d \omega)=\int_{F} \int_{\Delta} \widetilde{M}(\omega, d x d t) P(d \omega),
$$

for $F \in \mathcal{F}_{s}, \Delta=B \times(s, u], B \in \mathfrak{B}_{\mathbb{X}}$. This can be proved, thanks to the tightness of the measure $m$, with similar arguments as the existence of the regular modification of conditional probabilities. We refer to [40] and we omit the details.

Remark 2.4. The result above may be put in relation with Theorem 1.5 in Cairoli and Walsh [6]. However, there are important differences as the fact that our result is achieved in the framework of measure theory and the fact that we use a predicable compensator.

Example 2.1. Suppose $\mathbb{X}:=\{1,2, \ldots\}$ and that $W_{t}(x), 0 \leq t \leq T(x \in \mathbb{X})$, are independent standard Brownian motions on $[0, T]$. Let $\mathbb{F}$ be the $P$ augmented filtration generated by the Brownian motions. Then $\mu$ defined by

$$
\mu(\Delta):=\sum_{x=1}^{\infty} \int_{0}^{T} \chi_{\Delta}(x, t) d W_{t}(x), \quad \Delta \in \mathfrak{B}_{\mathbb{X}} \times \mathfrak{B}_{[0, T]},
$$

is a martingale random field w.r.t. $\mathbb{F}$ with orthogonal values. In this case the measures $M, m$ are

$$
M(\Delta)=m(\Delta)=\sum_{x=1}^{\infty} \int_{0}^{T} \chi_{\Delta}(x, t) d t .
$$


Example 2.2. Suppose $\mathbb{X}:=\{1,2, \ldots\}$, that $W_{t}(x), 0 \leq t \leq T(x \in \mathbb{X})$ are independent standard Brownian motions on $[0, T]$. Let $\mathbb{F}$ be the $P$ augmented filtration generated by the Brownian motions. Let

$$
\eta_{t}(x)=\eta_{0}(x) e^{\sigma_{x} W_{t}(x)-\frac{1}{2} \sigma_{x}^{2} t}, \quad 0 \leq t \leq T(x \in \mathbb{X}) .
$$

Define

$$
\mu(\Delta):=\sum_{x=1}^{\infty} \int_{0}^{T} \chi_{\Delta}(x, t) d \eta_{t}(x), \quad \Delta \in \mathfrak{B}_{\mathbb{X}} \times \mathfrak{B}_{[0, T]} .
$$

The measure $\mu$ given by (2.16) is an orthogonal martingale random field w.r.t. $\mathbb{F}$. In this case the measures $M, m$ are

$$
M(\Delta)=\sum_{x=1}^{\infty} \sigma_{x}^{2} \int_{0}^{T} \chi_{\Delta}(x, t) \eta_{t}^{2}(x) d t
$$

and

$$
m(\Delta)=\sum_{x=1}^{\infty} \sigma_{x}^{2} \eta_{0}^{2}(x) \int_{0}^{T} \chi_{\Delta}(x, t) e^{\sigma_{x}^{2} t} d t
$$

Example 2.3. Suppose $\mathbb{X}:=\{1,2, \ldots\}$ and that $\eta_{t}(x), 0 \leq t \leq T(x \in \mathbb{X})$, are independent square integrable Lévy martingales on $[0, T]$, i.e., for every $x, \eta_{t}(x), 0 \leq t \leq T$, is a Lévy process with characteristic exponent

$$
\psi_{x}(\lambda)=-\frac{1}{2} \sigma_{x}^{2} \lambda^{2}+i \gamma_{x} \lambda+\int_{\mathbb{R}}\left(e^{i \lambda v}-1-i \lambda v \chi_{[-1,1]}(v)\right) J_{x}(d v)
$$

with $\sigma_{x}^{2} \geq 0, \gamma_{x} \in \mathbb{R}$ and $J_{x}$ is a $\sigma$-finite Borel measure on $\mathbb{R} \backslash\{0\}$ such that

$$
\int_{\mathbb{R}} v^{2} J_{x}(d v)<\infty \text { and } \gamma_{x}+\int_{|v|>1} v J_{x}(d v)=0 \quad(x \in \mathbb{X})
$$

(cf. e.g. [42]). Let $\mathbb{F}$ be the $P$-augmented filtration generated by $\eta_{t}(x)$, $0 \leq t \leq T, x \in \mathbb{X}$. Then $\mu$ defined as in (2.16) by

$$
\mu(\Delta):=\sum_{x=1}^{\infty} \int_{0}^{T} \chi_{\Delta}(x, t) d \eta_{t}(x), \quad \Delta \in \mathfrak{B}_{\mathbb{X}} \times \mathfrak{B}_{[0, T]},
$$

is a martingale random field (w.r.t. $\mathbb{F}$ ) with orthogonal values. The measures $M, m$ are

$$
M(\Delta)=m(\Delta)=\sum_{x=1}^{\infty} \int_{0}^{T} \chi_{\Delta}(x, t)\left(\sigma_{x}^{2}+\int_{\mathbb{R}} v^{2} J_{x}(d v)\right) d t .
$$


Example 2.4. Suppose $\mathbb{V}:=\mathbb{R} \backslash\{0\}$ and consider the Poisson random measure

$$
N(A), \quad A \in \mathfrak{B}_{\mathbb{V}} \times \mathfrak{B}_{[0, T]} \quad(N(\mathbb{V} \times\{0\})=0),
$$

i.e. for every $A \in \mathfrak{B}_{\mathbb{V}} \times \mathfrak{B}_{[0, T]}$, the distribution of $N(A)$ satisfies

$$
E\left[e^{i \lambda N(A)}\right]=e^{\nu(A)\left(e^{i \lambda}-1\right)},
$$

where we have set

$$
\nu(A):=E[N(A)], \quad A \in \mathfrak{B}_{\mathbb{V}} \times \mathfrak{B}_{[0, T]} .
$$

The set-function $\nu$ represents a $\sigma$-finite measure on $\mathfrak{B}_{\mathbb{V}} \times \mathfrak{B}_{[0, T]}$. The random measure

$$
\widetilde{N}(A):=N(A)-\nu(A), \quad A \in \mathfrak{B}_{\mathbb{V}} \times \mathfrak{B}_{[0, T]},
$$

is the so-called compensated Poisson random measure. Let $\mathbb{F}$ be the $P$ augmented filtration of the $\sigma$-algebras generated by the values

$$
N(A), \quad A \in \mathfrak{B}_{\mathbb{V}} \times \mathfrak{B}_{[0, t]} \quad(0 \leq t \leq T) .
$$

Then the compensated Poisson random measure $\tilde{N}$ is a martingale random field (w.r.t. $\mathbb{F}$ ) with orthogonal values. In this case the corresponding conditional variance and variance measures are

$$
M_{N}(A)=m_{N}(A)=\int_{\mathbb{V} \times[0, T]} \chi_{A}(v, t) \nu(d v d t) .
$$

The compensated Poisson random measures appear naturally in the context of Example 2.3. In fact, for every $x \in \mathbb{X}$, the number of jumps of magnitude in $B \in \mathfrak{B}_{\mathbb{V}}$ made by the Lévy martingale $\eta_{t}(x), 0 \leq t \leq T$, in the time interval $(s, u]$ is represented by the Poisson random variable $N_{x}(B \times(s, u])$. The values

$$
N_{x}(B \times(s, u]), \quad B \in \mathfrak{B}_{\mathbb{V}}, \quad 0 \leq s<u \leq T,
$$

characterize a Poisson random measure (cf. (2.18))

$$
N_{x}(A), \quad A \in \mathfrak{B}_{\mathbb{V}} \times \mathfrak{B}_{[0, T]},
$$

with $\nu_{x}(A)=E\left[N_{x}(A)\right]$. Note that the measure $\nu_{x}(d v d t)$ admits a product representation in the form

$$
\nu_{x}(d v d t)=J_{x}(d v) \times d t
$$

where $J_{x}(d v)$ is the so-called Lévy measure of $\eta_{x}$ - cf. Example 2.3. Recall that

$$
J_{x}(B)=E\left[N_{x}(B \times(0,1]], \quad B \in \mathfrak{B}_{\mathbb{V}} .\right.
$$


See e.g. [42] for details. The Itô decomposition theorem shows that

$$
\eta_{t}(x)=\sigma_{x} W_{t}(x)+\int_{\mathbb{V} \times(0, t]} v \widetilde{N}_{x}(d v d t), \quad 0 \leq t \leq T,
$$

where $W_{t}(x), \widetilde{N}_{x}(d v d t)=N_{x}(d v d t)-\nu_{x}(d v d t), v \in \mathbb{V}, 0 \leq t \leq T(x \in \mathbb{X})$ are independent. Hence the set-function (2.17) admits representation as

$$
\mu(\Delta)=\sum_{x=1}^{\infty} \int_{[0, T]} \chi_{\Delta}(x, t) \sigma_{x} d W_{t}(x)+\sum_{x=1}^{\infty} \int_{\mathbb{V} \times[0, T]} \chi_{\Delta}(x, t) v \widetilde{N}_{x}(d v d t)
$$

on $\Delta \in \mathfrak{B}_{\mathbb{X}} \times \mathfrak{B}_{[0, T]}$. The construction above leads also to the definition of

$$
\begin{aligned}
\rho(G \times \Delta):= & \sum_{x=1}^{\infty} \int_{\{0\} \times[0, T]} \chi_{G \times \Delta}(v, x, t) \sigma_{x} d W_{t}(x) d \delta_{0}(d v) \\
& +\sum_{x=1}^{\infty} \int_{\mathbb{V} \times[0, T]} \chi_{G \times \Delta}(v, x, t) v \widetilde{N}_{x}(d v d t)
\end{aligned}
$$

on $G \in \mathfrak{B}_{\mathbb{R}}$ and $\Delta \in \mathfrak{B}_{\mathbb{X}} \times \mathfrak{B}_{[0, T]}$, which extends to a set-function $\rho(\Gamma)$, $\Gamma \in \mathfrak{B}_{\mathbb{R}} \times \mathfrak{B}_{\mathbb{X}} \times \mathfrak{B}_{[0, T]}$, as and example of Gaussian-Poisson mixture, see e.g. [19].

\section{Martingale random fields: non-anticipating in- tegration and differentiation}

\subsection{Non-anticipating integration}

We give a short review of the basic elements of the stochastic integration with respect to $\mu$ as integrator according to the classical Itô non-anticipating integraton scheme. This section introduces the grounds for the study of the non-anticipating differentiation.

We formalize the concept of partitions. Being $m$ a $\sigma$-finite measure on $\mathbb{X} \times[0, T]$, it is always possible to select an increasing sequence $\left(A_{n}\right)_{n}$ of sets $A_{n} \in \mathfrak{B}_{\mathbb{X}} \times \mathfrak{B}_{[0, T]}$ such that $m\left(A_{n}\right)<\infty$ and $\mathbb{X} \times[0, T]=\bigcup_{n=1}^{\infty} A_{n}$. Recall that $m(\mathbb{X} \times\{0\})=0$. In view of separability of $\mathbb{X} \times[0, T]$ generated by a product of semi-rings we can choose the sets $A_{n}$ of the form:

$$
A_{n}=\bigsqcup_{k=1}^{K_{n}} \Delta_{n k}
$$

where the sets

$$
\Delta_{n k}:=B_{n k} \times\left(s_{n k}, u_{n k}\right], \quad k=1, \ldots, K_{n},
$$

with $B_{n k} \in \mathfrak{B}$ and $0 \leq s_{n k}<u_{n k} \leq T$, are pairwise disjoint and constitute a generating semi-ring for $\mathfrak{B}_{\mathbb{X}} \times \mathfrak{B}_{[0, T]}$ (see also Remark 2.2). 
Definition 3.1. Denote $\mathcal{A}_{n}$ the family of sets (3.1). The family $\mathcal{A}_{n}$ is called a partition in $\mathbb{X} \times[0, T]$ with order of refinement $n$. We consider the partitions with increasing order of refinement such that

- Any element $\Delta_{n k}$ in $\mathcal{A}_{n}$ can be represented as finite union of elements in $\mathcal{A}_{n+1}$, i.e. $\Delta_{n k}=\bigsqcup_{j} \Delta_{n+1, j}$

- For $n \rightarrow \infty$ we have

$$
\max _{k=1, \ldots, K_{n}} m\left(\Delta_{n k}\right) \longrightarrow 0 \quad \text { and } \max _{k=1, \ldots, K_{n}}\left(u_{n k}-s_{n k}\right) \longrightarrow 0 .
$$

Then we call $\left(\mathcal{A}_{n}\right)_{n}$ a sequence of partitions in $\mathbb{X} \times[0, T]$.

Definition 3.2. A measurable function

$$
\phi: \Omega \times \mathbb{X} \times[0, T] \longrightarrow \mathbb{R}
$$

is a simple integrand if it admits the following representation

$$
\phi(x, t)=\sum_{k=1}^{K} \varphi_{k} \chi_{\Delta_{k}}(x, t),
$$

where $\Delta_{1}, \ldots, \Delta_{K}$ are pairwise disjoint sets of the form $\Delta_{k}=B_{k} \times\left(s_{k}, u_{k}\right]$ with $m\left(\Delta_{k}\right)<\infty$, and the values $\varphi_{k}$ are $\mathcal{F}_{s_{k}}$-measurable random variables satisfying

$$
E\left[\varphi_{k}^{2} M\left(\Delta_{k}\right)\right]<\infty .
$$

Thus the simple integrands are elements of

$$
L_{2}(P \times M):=L_{2}\left(\Omega \times \mathbb{X} \times[0, T], \mathcal{F} \times \mathfrak{B}_{\mathbb{X}} \times \mathfrak{B}_{[0, T]}, P \times M\right)
$$

with the finite norm given by

$$
\|\phi\|_{L_{2}(P \times M)}:=\left(E\left[\int_{\mathbb{X} \times[0, T]} \phi^{2}(x, t) M(d x d t)\right]\right)^{1 / 2} .
$$

Note that a simple integrand is a predictable function, i.e. it is measurable w.r.t. $\mathfrak{P}$.

The stochastic integral with respect to $\mu$ is well-defined on simple integrands. Namely, we have

$$
\mathfrak{J}(\phi)=\int_{\mathbb{X} \times[0, T]} \phi(x, t) \mu(d x d t):=\sum_{k=1}^{K} \varphi_{k} \mu\left(\Delta_{k}\right) .
$$

Moreover, the Itô isometry holds:

$$
\|\mathfrak{J}(\phi)\|_{L_{2}(P)}=\|\phi\|_{L_{2}(P \times M)} .
$$


Definition 3.3. A measurable function

$$
\phi: \Omega \times \mathbb{X} \times[0, T] \longrightarrow \mathbb{R}
$$

is a (general) integrand if it can be represented as a limit $\phi=\lim _{n \rightarrow \infty} \phi_{n}$ with convergence in $L_{2}(P \times M)$ of a sequence $\left(\phi_{n}\right)$ of simple integrands.

Remark 3.1. The set of general integrands corresponds to

$$
L_{2}(\mathfrak{P}):=L_{2}(\Omega \times \mathbb{X} \times[0, T], \mathfrak{P}, P \times M),
$$

that is the subspace of elements in $L_{2}(P \times M)$ admitting a predictable representative. To explain, as the simple integrands are predictable, so are their pointwise limits $P \times M$-a.e. A general integrand is the limit $\phi=$ $\lim _{n \rightarrow \infty} \phi_{n}$ in $L_{2}(P \times M)$ of some simple integrands $\left(\phi_{n}\right)_{n}$. We can define the predictable function $\widetilde{\phi}$ as the pointwise limit $\widetilde{\phi}(\omega, x, t)=\lim _{j \rightarrow \infty} \phi_{n_{j}}(\omega, x, t)$ of a subsequence $\left(\phi_{n_{j}}\right)_{j}$ for all the points $(\omega, x, t)$ where the limits exists and it can be set $\widetilde{\phi}(\omega, x, t)=0$ elsewhere. We can then see that $\widetilde{\phi}$ is a modification of $\phi$. Conversely, any predictable function in $L_{2}(P \times M)$ can be approximated $P \times M$-a.e. by a linear combination of indicators

$$
\chi_{F \times B \times(s, u]}=\chi_{F} \chi_{B \times(s, u]}
$$

where $0 \leq s<u \leq T, B \in \mathfrak{B}$ and $F \in \mathcal{F}_{s}$ - cf. (2.3). Since these sets consitute a semi-ring in $\mathfrak{P}$, these indicators constitute a complete system in $L_{2}(\mathfrak{P})$. Thus any element in $L_{2}(\mathfrak{P})$ represents an integrand.

In the following result we characterize explicitly a sequence of simple integrands $\left(\phi_{n}\right)_{n}$ approximating the given element $\phi \in L_{2}(\mathfrak{P})$. This completes the remark aforementioned.

Lemma 3.1. Let $\phi \in L_{2}(\mathfrak{P})$. For any sequence of partitions $\left(\mathcal{A}_{n}\right)_{n}$ in $\mathbb{X} \times(0, T]$, we can define the simple integrands $\phi_{n}(x, t),(x, t) \in \mathbb{X} \times[0, T]$, as

$$
\phi_{n}(x, t)=\sum_{k=1}^{K_{n}} E\left[\frac{\int_{\Delta_{n k}} \phi(x, t) M(d x d s)}{E\left[M\left(\Delta_{n k}\right) \mid \mathcal{F}_{s_{n k}}\right]} \mid \mathcal{F}_{s_{n k}}\right] \chi_{\Delta_{n k}}(x, t)
$$

where $\Delta_{n k}=B_{n k} \times\left(s_{n k}, u_{n k}\right], k=1, \ldots, K_{n}$, are the elements in $\mathcal{A}_{n}$. The sequence $\left(\phi_{n}\right)_{n}$ approximates $\phi$ in $L_{2}(P \times M)$.

Proof. Denote by $\left(\theta_{n}\right)_{n}$ a sequence of simple integrands:

$$
\theta_{n}(x, t)=\sum_{k=1}^{K_{n}} \vartheta_{k} \chi_{\Delta_{n k}}(x, t)
$$


approximating $\phi$ in $L^{2}(P \times M)$ - see Remark 3.1. With no loss of generality we can represent $\theta_{n}$ and $\phi_{n}$ on the elements of $\mathcal{A}_{n}$. Consider

$$
\begin{aligned}
\left\|\phi_{n}-\theta_{n}\right\|_{L_{2}(P \times M)} & =\sum_{k=1}^{K_{n}} E\left[\left(\frac{E\left[\int_{\Delta_{n k}} \phi(x, t) M(d x d t) \mid \mathcal{F}_{s_{n k}}\right]}{E\left[M\left(\Delta_{n k}\right) \mid \mathcal{F}_{s_{n k}}\right]}-\vartheta_{n k}\right)^{2} M\left(\Delta_{n k}\right)\right] \\
& =\sum_{k=1}^{K_{n}} E\left[\frac{E\left[\int_{\Delta_{n k}}\left(\phi(x, t)-\vartheta_{n k}\right) M(d x d t) \mid \mathcal{F}_{s_{n k}}\right]^{2}}{E\left[M\left(\Delta_{n k}\right) \mid \mathcal{F}_{s_{n k}}\right]}\right] \\
& \leq \sum_{k=1}^{K_{n}} E\left[\frac{E\left[\left(\int_{\Delta_{n k}}\left(\phi(x, t)-\vartheta_{n k}\right) M(d x d t)\right)^{2} \mid \mathcal{F}_{s_{n k}}\right]}{E\left[M\left(\Delta_{n k}\right) \mid \mathcal{F}_{s_{n k}}\right]}\right] \\
& \leq \sum_{k=1}^{K_{n}} E\left[E\left[\left(\int_{\Delta_{n k}}\left(\phi(x, t)-\vartheta_{n k}\right)^{2} M(d x d t) \mid \mathcal{F}_{s_{n k}}\right]\right]\right. \\
& =E\left[\int_{\mathbb{X} \times[0, T]}\left(\phi(x, t)-\vartheta_{n k}\right)^{2} M(d x d t)\right] \\
& =\left\|\phi-\theta_{n}\right\|_{L_{2}(P \times M)}
\end{aligned}
$$

by application of the Hölder inequality for conditional expectations. Hence

$$
\left\|\phi-\phi_{n}\right\|_{L_{2}(P \times M)} \leq 2\left\|\phi-\theta_{n}\right\|_{L_{2}(P \times M)} \stackrel{n \rightarrow \infty}{\longrightarrow} 0 .
$$

According to classical Itô integration scheme, for any integrand $\phi=\lim _{n \rightarrow \infty} \phi_{n}$ we can define the non-anticipating integral as the limit

$$
\mathfrak{J}(\phi)=\int_{\mathbb{X} \times[0, T]} \phi(x, t) \mu(d x d t):=\lim _{n \rightarrow \infty} \int_{\mathbb{X} \times[0, T]} \phi_{n}(x, t) \mu(d x d t)
$$

with convergence in $L_{2}(P)$. Moreover, the basic rules of calculus hold:

$$
E\left[\int_{\mathbb{X} \times(t, T]} \phi(x, s) \mu(d x d s) \mid \mathcal{F}_{t}\right]=0
$$

and

$$
\begin{aligned}
E\left[\int_{\mathbb{X} \times(t, T]} \phi(x, s) \mu(d x d s)\right. & \left.\int_{\mathbb{X} \times(t, T]} \theta(x, s) \mu(d x d s) \mid \mathcal{F}_{t}\right] \\
& =E\left[\int_{\mathbb{X} \times(t, T]} \phi(x, s) \theta(x, s) M(d x d s) \mid \mathcal{F}_{t}\right] .
\end{aligned}
$$

Remark 3.2. Let $\phi \in L^{2}(\mathfrak{P})$. For any $\Delta \in \mathfrak{B}_{\mathbb{X}} \times \mathfrak{B}_{[0, T]}$, let us define

$$
\mathfrak{J}(\phi, \Delta):=\int_{\Delta} \phi(x, t) \mu(d x d t) .
$$


From (3.6) and (3.7) we see that the set-function $\mathfrak{J}(\phi, \Delta), \Delta \in \mathfrak{B}_{\mathbb{X}} \times \mathfrak{B}_{[0, T]}$, is a martingale random field (see Definition 2.1) with corresponding conditional variance and variance measures given by

$$
M(\phi, \Delta)=\int_{\Delta} \phi^{2}(x, t) M(d x d t)
$$

and

$$
m(\phi, \Delta)=E\left[\int_{\Delta} \phi^{2}(x, t) M(d x d t)\right] .
$$

Thus martingale random fields appear naturally after non-anticipating integration with respect to another martingale random field as integrator.

\section{$3.2 \quad$ Non-anticipating stochastic derivative}

The non-anticipating integral $\mathfrak{J}$ can be presented in other words as being the isometric linear operator

$$
\mathfrak{J}: L^{2}(\mathfrak{P}) \Longrightarrow L^{2}(P)
$$

and the integration can be carried out via the limit (3.5) with the use of the integrands given in (3.4).

Definition 3.4. Let $\mathfrak{D}=\mathfrak{J}^{*}$ be the adjoint linear operator to the nonanticipating integral:

$$
\mathfrak{D}: L^{2}(P) \Longrightarrow L^{2}(\mathfrak{P}) \text {. }
$$

we call $\mathfrak{D}$ the non-anticipating derivative.

The non-anticipating derivative was first introduced in [18] for the Brownian motion as integrator and the proofs exploited widely its properties. In [14] the derivative with respect to martingale processes was introduced. In [13] the first extension to random fields is considered, considering only random fields with independent values. Such are the random fields in the examples of Section 2. Note that the random fields generated by integration, see Remark 3.2, do not have, in general, independent values. Still in the framework of random fields with independent values, [15] provides some rules of calculus for the non-anticipating derivative alternative to the differentiation rule given in Theorem 3.1 below. Having introduced the concept of martingale random fields as in Definition 2.1, we see that the non-anticipating derivative can be extended to this framework.

Theorem 3.1. The non-anticipating derivative is well-defined for all the elements $\xi \in L_{2}(P)$. The derivative $\mathfrak{D} \xi$ is given by the limit

$$
\mathfrak{D} \xi=\lim _{n \rightarrow \infty} \varphi_{n}, \quad \text { i.e. } \quad\|\mathfrak{D} \xi-\varphi\|_{L_{2}(P \times M)} \stackrel{n \rightarrow \infty}{\longrightarrow} 0,
$$


of the simple integrands

$$
\varphi_{n}(x, t):=\sum_{k=1}^{K_{n}} E\left[\xi \frac{\mu\left(\Delta_{n k}\right)}{E\left[M\left(\Delta_{n k}\right) \mid \mathcal{F}_{s_{n k}}\right]} \mid \mathcal{F}_{s_{n k}}\right] \chi_{\Delta_{n k}}(x, t), \quad(x, t) \in \mathbb{X} \times[0, T],
$$

where the sets $\Delta_{n k}$ are the elements of $\mathcal{A}_{n}$ - cf. Definition 3.1. Moreover, the following stochastic integral representation holds:

$$
\xi=\xi^{(0)} \oplus \int_{\mathbb{X} \times[0, T]} \mathfrak{D}_{x, t} \xi \mu(d x d t)
$$

where $\xi^{(0)} \in L_{2}(P)$ such that $\mathfrak{D}_{x, t} \xi^{(0)} \equiv 0$.

According to the Kunita-Watanabe decompostition (see also Föllmer-Schweizer decomposition) in our framework, for any $\xi \in L_{2}(P)$ there exists an integrand $\varphi$ such that

$$
\xi=\xi^{(0)} \oplus \int_{\mathbb{X} \times[0, T]} \varphi \mu(d x d t) .
$$

The novelty of (3.10) is the characterization of this integrand in terms of the very variable $\xi$ and the integrator $\mu$ as the only given data. In this sense the representation (3.10) springs from the same motivation as the well-known Clark-Ocone formula, being at the same time substantially different. The Clark-Ocone formula has at its core the Malliavin derivative operator which can be regarded as the adjoint to the Skorohod integral and belongs to forms of stochastic calculus that do not necessarily take information (i.e. the filtration $\mathbb{F}$ ) into account. The Clark-Ocone formula was initially tailored for the Brownian motion as integrator (see e.g. [35], [37]), and later on generalized to Poisson processes, Poisson random measures and Lévy processes (see e.g. [1], [34], [33], [38] and [17]). Only recently it has been extended to the frame of integration with respect to stochastic measures with independent values on a space-time product (see e.g. [15]). The main difficulty in the extension of the Clark-Ocone formula has been the extension of the definition of the Malliavin derivative with respect to more general types of random measures. On the other side the very use of the Malliavin derivative implies the restriction of the use of this formula to the domain of this operator which is strictly included in $L_{2}(P)$. Using techniques of white noise analysis one can extend such a domain to the whole $L_{2}(P)$. This has been done for the Brownian setting and the Poisson random measures, see, e.g. [16], [17]. We would like to remark that formula (3.10) above is well-defined for all elements $\xi \in L_{2}(P)$.

Remark 3.3. Note that for any $\xi$ of the form

$$
\xi=\int_{\mathbb{X} \times[0, T]} \varphi(x, t) \mu(d x d t)
$$


for some $\varphi \in L_{2}(\mathfrak{P})$, we clearly have $\mathfrak{D} \xi \equiv \varphi$. In fact, for any $\Delta=B \times(s, u]$,

$$
\begin{aligned}
E\left[\xi \frac{\mu(\Delta)}{E\left[M(\Delta) \mid \mathcal{F}_{s}\right]} \mid \mathcal{F}_{s}\right] & =E\left[\int_{\Delta} \varphi(x, t) \mu(d x d t) \frac{\mu(\Delta)}{E\left[M(\Delta) \mid \mathcal{F}_{s}\right]} \mid \mathcal{F}_{s}\right] \\
& =E\left[\frac{\int_{\Delta} \varphi(x, t) M(d x d t)}{E\left[M(\Delta) \mid \mathcal{F}_{s}\right]} \mid \mathcal{F}_{s}\right]
\end{aligned}
$$

which is the approximation given in Lemma 3.1.

Remark 3.4. Note that the non-anticipating derivative is continuous in the sense that

$$
\xi=\lim _{n \rightarrow \infty} \xi_{n}, \quad \text { i.e. } \quad\left\|\xi-\xi_{n}\right\|_{L_{2}(P)} \stackrel{n \rightarrow \infty}{\longrightarrow} 0
$$

implies

$$
\mathfrak{D} \xi=\lim _{n \rightarrow \infty} \mathfrak{D} \xi_{n}, \quad \text { i.e. } \quad\left\|\mathfrak{D} \xi-\mathfrak{D} \xi_{n}\right\|_{L_{2}(P \times M)} \stackrel{n \rightarrow \infty}{\longrightarrow} 0 .
$$

Proof of Theorem 3.1. The proof follows arguments in the same line of [14]. Here we only sketch the fundamental steps. Let $\mathcal{H} \subseteq L_{2}(P)$ be the subspace of all stochastic integrals with respect to $\mu$. For any $\Delta_{n k}=B_{n k} \times\left(s_{n k}, u_{n k}\right]$ of the partition $\mathcal{A}_{n}$ of level of refinement $n$, let $\mathcal{H}\left(\Delta_{n k}\right) \subseteq L_{2}(P)$ be the subspace all random variables of form $\xi=\psi \mu\left(\Delta_{n k}\right)$ where $\psi$ is $\mathcal{F}_{s_{n k}}$-measurable. Then

$$
\mathcal{H}=\lim _{n \rightarrow \infty} \sum_{k=1}^{K_{n}}{ }_{\oplus} \mathcal{H}\left(\Delta_{n k}\right) .
$$

Let $\xi \in L_{2}(P)$, then its projection on $\mathcal{H}\left(\Delta_{n k}\right)$ is given by

$$
E\left[\xi \frac{\mu\left(\Delta_{n k}\right)}{E\left[M\left(\Delta_{n k}\right) \mid \mathcal{F}_{s_{n k}}\right]} \mid \mathcal{F}_{s_{n k}}\right] \mu\left(\Delta_{n k}\right) .
$$

Hence the projection on $\sum_{k=1}^{K_{n}} \oplus \mathcal{H}\left(\Delta_{n k}\right)$ is

$$
\begin{aligned}
\widehat{\xi}_{n} & :=\sum_{k=1}^{K_{n}} E\left[\xi \frac{\mu\left(\Delta_{n k}\right)}{E\left[M\left(\Delta_{n k} \mid \mathcal{F}_{s_{n k}}\right]\right.} \mid \mathcal{F}_{s_{n k}}\right] \mu\left(\Delta_{n k}\right) \\
& =\int_{\mathbb{X} \times[0, T]} \varphi_{n}(x, t) \mu(d x d t),
\end{aligned}
$$

with $\varphi_{n}$ given by (3.9). Of course the projection $\widehat{\xi}$ of $\xi$ onto $\mathcal{H}$ is given by

$$
\widehat{\xi}:=\lim _{n \rightarrow \infty} \widehat{\xi}_{n}=\lim _{n \rightarrow \infty} \int_{\mathbb{X} \times[0, T]} \varphi_{n}(x, t) \mu(d x d t) .
$$

Set $\varphi:=\lim _{n \rightarrow \infty} \varphi_{n}$ in $L_{2}(P \times M)$, then

$$
\widehat{\xi}=\int_{\mathbb{X} \times[0, T]} \varphi(x, t) \mu(d x d t)
$$

- see Remark 3.3. Clearly,

$$
\xi^{(0)}:=\xi \ominus \widehat{\xi}
$$

and, from (3.7), we se that $\varphi=\mathfrak{J}^{*} \xi$, then $\mathfrak{D} \xi=\varphi$. Naturally, $\mathfrak{D} \xi^{(0)} \equiv 0$. 


\section{The large markets, minimal variance hedging and completeness}

In a large market setting, we consider the risky assets to be indexed by the topological space $\mathbb{X}$ (cf. Section 2). This interpretation is consistent with the "small" market setting where $\mathbb{X}=\{1, \ldots, N\}$ and with the "countable" market setting studied e.g. in [7], [8] where $\mathbb{X}:=\{1,2, \ldots\}$. In both these cases we can consider the discrete topology. In the general market model we are describing, $\mathbb{X}$ does not need to be discrete. Then if $x \in \mathbb{X}$ denotes the single security, a set $B \in \mathfrak{B}_{\mathbb{X}}$ is interpreted as a group of securities which we call package $B$. In this line, if $S_{t}(x), 0 \leq t \leq T$, is the price of a single security, we denote by $S_{t}(B), 0 \leq t \leq T$, the price of the package $B$. The prices are $\mathbb{F}$-adapted processes where $\mathbb{F}$ is the filtration corresponding to the flow of information available in time - cf. (2.1). Following the intuition given by the small markets, we consider prices to be additive in the sense that

$$
S_{t}(B)=S_{t}\left(B_{1}\right)+S_{t}\left(B_{2}\right)
$$

for $B=B_{1} \bigsqcup B_{2} \in \mathfrak{B}_{\mathbb{X}}, B_{1} \cap B_{2}=\emptyset$. For example, consider $B_{1}=\left\{x_{1}\right\}, B_{2}=$ $\left\{x_{2}\right\}$ with $x_{1} \neq x_{2}$ and $B=\left\{x_{1}, x_{2}\right\}$. Let $R_{t}, 0 \leq t \leq T$, be the price process of a reference riskless asset (money market account) with dynamics given by

$$
d R_{t}=R_{t} r_{t} d t ; \quad R_{0}=1 .
$$

The instantaneous interest rate $r_{t}, 0 \leq t \leq T$, is a positive predictable process.

In this market $\mu$, defined by

$$
\mu(B \times(s, u]):=\frac{S_{u}(B)}{R_{u}}-\frac{S_{s}(B)}{R_{s}},
$$

is interpreted as the excessive return associated with holding the securities package $B$ over the time period $(s, u]$. We assume that

- $\mathbb{X} \times(0, T]$ can be generated by a semi-ring of sets of the form (2.2) for which

$$
E\left[\mu^{2}(B \times(s, u])\right]<\infty,
$$

- the conditional expected values of the excessive returns associated with these packages and periods are zero, i.e.

$$
E\left[\mu(B \times(s, u]) \mid \mathcal{F}_{s}\right]=0,
$$

- the variance $m(B \times(s, u])=E\left[\mu^{2}(B \times(s, u])\right]$, which extends to a $\sigma$-finite Borel measure $m(d x d t),(x, t) \in \mathbb{X} \times[0, T]$, is tight $^{2}$

\footnotetext{
${ }^{2}$ From the point of view of applications, this is not a strong assumption in fact we recall that any $\sigma$-finite Borel measure on a complete separable metric space is tight.
} 
- and the excessive returns associated with disjoint securities packages are conditionally non-correlated, i.e.

$$
E\left[\mu\left(B_{1} \times(s, u]\right) \mu\left(B_{2} \times(s, u]\right) \mid \mathcal{F}_{s}\right]=0, \quad B_{1} \cap B_{2}=\emptyset,
$$

then, with $\mu(B \times\{0\}) \equiv 0, \mu$ admits extension

$$
\mu(\Delta), \quad \Delta \in \mathfrak{B}_{\mathbb{X}} \times \mathfrak{B}_{[0, T]},
$$

as a martingale random field, see Definition 2.1. The properties of additivity, adaptedness, martingality and orthogonality of $\mu$ follow readily from the definition and the assumptions above.

Remark 4.1. To this end it is sufficient that for any $B \in \mathfrak{B}$ the excessive return process $\eta(B)$ given by

$$
\eta_{t}(B):=\frac{S_{t}(B)}{R_{t}}-\frac{S_{0}(B)}{R_{0}}, \quad 0 \leq t \leq T,
$$

is a square integrable martingale, which means that the probability measure $P$ is risk neutral, and that the excessive return processes associated with distinct packages of securities have conditionally non-correlated increments, i.e.

$$
E\left[\left(\eta_{u}\left(B_{1}\right)-\eta_{s}\left(B_{1}\right)\right)\left(\eta_{u}\left(B_{2}\right)-\eta_{s}\left(B_{2}\right)\right) \mid \mathcal{F}_{s}\right]=0, \quad s \leq u, B_{1} \cap B_{2}=\emptyset .
$$

Note that in this case the measure $m$ is not merely $\sigma$-finite on $\mathfrak{B}_{\mathbb{X}} \times \mathfrak{B}_{[0, T]}$, but $m(\cdot \times[0, T])$ is a $\sigma$-finite measure on $\mathbb{X}$. This will be the case for the markets studied in Section 5 .

In this large market with $\mathbb{X}$ securities available a trading strategy will be characterized by a stochastic function $\phi \in L_{2}(\mathfrak{P})$ and an initial $\mathcal{F}_{0}$-measurable endowment $w$. At any time $0 \leq t \leq T$, the stochastic function $\phi(x, t)$, $x \in \mathbb{X}$, represents the holdings in the security $x$. Correspondingly, for any $t$, we denote by

$$
\phi_{B}(x, t):=\phi(x, t) \chi_{B}(x), \quad x \in \mathbb{X},
$$

the function describing the holdings in the package $B$. We will call $\phi$, the density of investments.

The following argument gives a motivation for the use of the density of investments. Let us consider a finite number of investment possibilities in the pairwise disjont packages $B_{1}, \ldots, B_{K} \in \mathfrak{B}$ (note that this would be the situation in a small market) and a discrete-time trading situation with $0=s_{1}<u_{1} \leq s_{2}<\ldots<u_{J}=T$. We can consider holdings in each package $B_{k}$ of the form:

$$
\phi_{B_{k}}(x, t)=\sum_{j=1}^{J} \phi_{k j} \chi_{\left(s_{j}, u_{j}\right]}(t), \quad 0 \leq t \leq T,
$$


where the values $\phi_{k j}$ are $\mathcal{F}_{s_{j}}$-measurable random variables and represent a uniform amount of holdings over the packages $B_{k}$. We assume

$$
E\left[\sum_{k=1}^{K} \int_{\mathbb{X} \times[0, T]} R_{t}^{2} \phi_{B_{k}}^{2}(x, t) \chi_{B_{k}}(x) M(d x d t)\right]<\infty .
$$

Hence the density of investments $\phi(x, t),(x, t) \in \mathbb{X} \times[0, T]$, is given by

$$
\phi(x, t)=\sum_{k=1}^{K} \phi_{B_{k}}(x, t) \chi_{B_{k}}(x)=\sum_{k=1}^{K} \sum_{j=1}^{J} \phi_{k j} \chi_{B_{k} \times\left(s_{j}, u_{j}\right]}(x, t)
$$

and is an element of $L_{2}(\mathfrak{P})$. The corresponding value process $\xi_{t}, 0 \leq t \leq T$ $\left(\xi_{0}=w\right)$, of the self-financing strategy is given by

$$
\begin{aligned}
d \xi_{t} & =\left[\xi_{t}-\sum_{k=1}^{K} \phi_{B_{k}}(x, t) S_{t}\left(B_{k}\right)\right] r_{t} d t+\sum_{k=1}^{K} \phi_{B_{k}}(x, t) d S_{t}\left(B_{k}\right) \\
& =\xi_{t} r_{t} d t+R_{t} \sum_{k=1}^{K} \phi_{B_{k}}(x, t) \mu_{t}\left(B_{k} \times d t\right) \\
& =\xi_{t} r_{t} d t+R_{t} \int_{\mathbb{X}} \phi(x, t) \mu(d x d t) .
\end{aligned}
$$

From (4.4) we can consider both the standard approximation that leads from discrete-time trading to continuous-time trading on $[0, T]$ and the approximation of square integrable functions via simple functions to extend the variety of investments possibility on $\mathbb{X}$. To combine the two, we take the partitions of $\mathbb{X} \times[0, T]$ into account as in Definition 3.1 where, for every $n$, the $B_{n k}, k=1, \ldots, K_{n}$, represent the packages available and $0=s_{n 1}<u_{n 1} \leq s_{n 2}<\ldots \leq u_{n K_{n}}=T$ are the trading times. We consider the convergence in $L_{2}(P \times M)$. The simple densities of investments of type (4.4) approximate the general ones $\phi(x, t),(x, t) \in \mathbb{X} \times[0, T]$. The approximation argument carries through in (4.5), thus given an initial endowment $w$ and a (self-financing) density of investment $\phi$, the corresponding value process is given by:

$$
d \xi_{t}=\xi_{t} r_{t} d t+R_{t} \int_{\mathbb{X}} \phi(x, t) \mu(d x d t), \quad \xi_{0}=w,
$$

where

$$
E\left[\int_{\mathbb{X} \times[0, T]} R_{t}^{2} \phi^{2}(x, t) M(d x d t)\right]<\infty .
$$

Clearly, the discounted value process $\bar{\xi}_{t}:=\frac{\xi_{t}}{R_{t}}, 0 \leq t \leq T$, is a martingale w.r.t. $\mathbb{F}$, in fact

$$
\bar{\xi}_{t}=w+\int_{\mathbb{X} \times(0, t]} \phi(x, s) \mu(d x d s), \quad 0 \leq t \leq T .
$$

Conversely, we have the following immediate result. 
Proposition 4.1. Let $\zeta_{t}, 0 \leq t \leq T$, be a martingale w.r.t. $\mathbb{F}$ such that $E\left[\zeta_{t}^{2}\right]<\infty$. If the random variable $\zeta_{T}$ admits stochastic integral representation

$$
\zeta_{T}=w+\int_{\mathbb{X} \times[0, T]} \varphi(x, t) \mu(d x d t) \quad\left(w=E\left[\zeta_{T}\right]\right)
$$

by means of some $\varphi \in L_{2}(\mathfrak{P})$, then the process $\zeta_{t}, 0 \leq t \leq T$, represents the discounted value process of the self-financing strategy with density of investments $\varphi$ and initial endowment $w$.

Proof. It is enough to consider

$$
\xi_{t}:=R_{t} \zeta_{t}=E\left[R_{t} \zeta_{T} \mid \mathcal{F}_{t}\right]=R_{t} w+R_{t} \int_{\mathbb{X} \times[0, T]} \varphi(x, t) \mu(d x d t),
$$

so we have

$$
d \xi_{t}=\xi_{t} r_{t} d t+R_{t} \int_{\mathbb{X}} \varphi(x, t) \mu(d x d t)
$$

and $\xi_{0}=w$.

\subsection{Market completeness}

Let $\mathcal{H} \subseteq L_{2}(P)$ be the space of all replicable claims. Namely, a claim with payoff $\xi$ belongs to $\mathcal{H}$ if there exists an $\mathcal{F}_{0}$-measurable $w$ and $\phi \in L_{2}(\mathfrak{P})$ such that the corresponding value process $\xi_{t}, 0 \leq t \leq T$, has final value $\xi_{T}=\xi$. From Proposition 4.1, we see that $\xi \in L_{2}(P)$ is replicable if and only if it admits representation in the form

$$
\xi=R_{T}\left(w+\int_{\mathbb{X} \times[0, T]} \varphi(x, t) \mu(d x d t)\right)
$$

with $w=E\left[\frac{\xi}{R_{T}}\right]$.

Definition 4.1. The market is complete if $\mathcal{H}=L_{2}(P)$.

Completeness of the market is linked to the possibility of giving integral representations to all random variables in $L_{2}(P)$ which represent claims in our setting. This depends on the martingale random field $\mu$ stemming out of the price models. Confining ourselves to random fields with independent values, a characterization of those fields for which $\mathcal{H}=L_{2}(P)$ is given in e.g. [19, Theorem 3.4], see also [13, Remark 4].

In [8] a study of completeness of large markets with $\mathbb{X}=\{1,2, \ldots\}$ is given. In particular the problem addressed is to characterize the relation between the completeness of the small markets $\mathbb{X}_{n}=\{1, \ldots, n\}$ versus the one of the large market $\mathbb{X}=\bigcup_{n} \mathbb{X}_{n}$. The following result is in the same line. 
Theorem 4.1. Suppose that $\mu$ is some orthogonal martingale random field that is generating the filtration $\mathbb{F}$ i.e.

$$
\mathcal{F}_{t}=\sigma\left\{\mu(B \times(s, u]) ; B \in \mathfrak{B}_{\mathbb{X}}, 0<s<u \leq t\right\}, \quad 0 \leq t \leq T
$$

and denote by $\mathbb{F}^{(B)}$ the filtration generated by $\mu$ "restricted to" $B \in \mathfrak{B}_{\mathbb{X}}$ i.e.

$$
\mathcal{F}_{t}^{(B)}:=\sigma\left\{\mu(A \times(s, u]) ; A \in \mathfrak{B}_{\mathbb{X}}, A \subseteq B, 0<s<u \leq t\right\}, \quad 0 \leq t \leq T .
$$

Denote by $\mathfrak{P}_{B}$ the $\sigma$-algebra generated by sets of the form

$$
F \times A \times(s, u], \quad F \in \mathcal{F}_{s}^{(B)}, A \in \mathfrak{B}_{\mathbb{X}}, A \subseteq B, 0 \leq s<u \leq T .
$$

If the market is complete, then for every $B \in \mathfrak{B}_{\mathbb{X}}$ and every $\xi \in L_{2}\left(\mathcal{F}_{T}^{(B)}\right)$ there exists some $\phi \in L_{2}\left(\mathfrak{P}_{B}\right)$ such that

$$
\int_{\mathbb{X} \times[0, T]} \phi(x, t) \mu(d x d t)=\xi-E[\xi]
$$

For the market to be complete, it is sufficient that there exists some sequence of sets $\left(B_{n}\right)_{n}$ with $\mathbb{X}=\bigcup_{\mathbb{N}} B_{n}$ such that for every $n$ and every $\xi \in L_{2}\left(\mathcal{F}_{T}^{\left(B_{n}\right)}\right)$ there exists some $\phi \in L_{2}\left(\mathfrak{P}_{B}\right)$ such that (4.7) holds.

Proof. Suppose that the market is complete and that $\xi \in L_{2}\left(\mathcal{F}_{T}^{(B)}\right)$ for some $B \in \mathfrak{B}_{\mathbb{X}}$. By Theorem $3.1 \xi-E[\xi]=\lim _{n \rightarrow \infty} \xi_{n}$ where

$$
\xi_{n}=\sum_{k=1}^{K_{n}} E\left[\xi \frac{\mu\left(\Delta_{n k}\right)}{E\left[M\left(\Delta_{n k}\right) \mid \mathcal{F}_{s_{n k}}\right]} \mid \mathcal{F}_{s_{n k}}\right] \mu\left(\Delta_{n k}\right)
$$

and $\left(\Delta_{n 1}, \ldots, \Delta_{n K_{n}}\right)_{\mathbb{N}}$ is a partition sequence, see Definition 3.1. Without loss of generality we assume that the $B_{n k}$ 's are contained in either $B$ or $B^{C}$ so that

$$
\begin{aligned}
\xi_{n}=\sum_{\substack{k=1 \\
B_{n k} \subseteq B}}^{K_{n}} E\left[\xi \frac{\mu\left(\Delta_{n k}\right)}{E\left[M\left(\Delta_{n k}\right) \mid \mathcal{F}_{s_{n k}}\right]} \mid \mathcal{F}_{s_{n k}}\right] \mu\left(\Delta_{n k}\right) \\
+\sum_{\substack{k=1 \\
B_{n k} \subseteq B}}^{K_{n}} E\left[\xi \frac{\mu\left(\Delta_{n k}\right)}{E\left[M\left(\Delta_{n k}\right) \mid \mathcal{F}_{s_{n k}}\right]} \mid \mathcal{F}_{s_{n k}}\right] \mu\left(\Delta_{n k}\right) .
\end{aligned}
$$

Noting that the first term is $\mathcal{F}_{T}^{(B)}$-measurable and that

$$
E\left[E\left[\xi \frac{\mu\left(\Delta_{n k}\right)}{E\left[M\left(\Delta_{n k} \mid \mathcal{F}_{s_{n k}}\right]\right.} \mid \mathcal{F}_{s_{n k}}\right] \mu\left(\Delta_{n k}\right) \mid \mathcal{F}_{T}^{(B)}\right]=0
$$


we have

$E\left[\xi_{n} \mid \mathcal{F}_{T}^{(B)}\right]=\sum_{\substack{k=1 \\ B_{n k} \subseteq B}}^{K_{n}} E\left[\xi \frac{\mu\left(\Delta_{n k}\right)}{E\left[M\left(\Delta_{n k} \mid \mathcal{F}_{s_{n k}}\right]\right.} \mid \mathcal{F}_{s_{n k}}\right] \mu\left(\Delta_{n k}\right)=\int_{\mathbb{X} \times[0, T]} \phi_{n} \mu(d x d s)$

where $\phi_{n} \in L_{2}\left(\mathfrak{P}_{B}\right)$ is a simple integrand. Moreover, as $\xi \in L_{2}\left(\mathcal{F}_{T}^{(B)}\right)$,

$$
E\left[\xi_{n} \mid \mathcal{F}_{T}^{(B)}\right] \stackrel{n \rightarrow \infty}{\longrightarrow} \xi-E[\xi]
$$

and (4.7) holds for some $\phi \in L_{2}\left(\mathfrak{P}_{B}\right)$ (cf. Remark 3.1).

For the latter part suppose that $\xi \in L_{2}(P)$. By assumption there exists some sequence $\left(\phi_{n}\right)$ of elements in $L_{2}(\mathfrak{P})$ such that

$$
\int_{B_{n} \times[0, T]} \phi_{n}(x, t) \mu(d x d t)=E\left[\xi \mid \mathcal{F}_{T}^{\left(B_{n}\right)}\right]-E[\xi] .
$$

As $\mathcal{F}=\bigvee_{\mathbb{N}} \mathcal{F}_{T}^{\left(B_{n}\right)}$, we have that for any $\xi \in L_{2}(P)$

$$
E\left[\xi \mid \mathcal{F}_{T}^{(B)}\right] \stackrel{n \rightarrow \infty}{\longrightarrow} \xi
$$

in the $L_{2}$-sense (cf. $\left.[25 \text {, Theorem } 7.23]^{3}\right)$. Hence there exists some $\phi \in L_{2}(\mathfrak{P})$ such that (4.7) holds - see Section 3.1.

Completeness in the case of a discrete $\mathbb{X}$ without the orthogonality assumption is treated in [8]: the last part of Theorem 4.1 is proved to hold in that setting (cf. [8, Proposition 3.15]), but the first part does not hold in general. It is however proved that any attainable claim in the large market can be approximated by a trading strategy based on a finite number of assets ([8, Theorem 5.1]).

\subsection{Minimal variance hedging}

Let us now turn the attention to the generally incomplete markets. Then a given claim $\xi \in L_{2}(P)$ may not be perfectly replicable, i.e. $\xi \notin \mathcal{H}$. Then the minimal variance hedging problem (see (1.1)) is to find an initial endowment $\widehat{w}$ and a density of investments $\widehat{\phi}$ such that the claim

$$
\widehat{\xi}:=R_{T}\left(\widehat{w}+\int_{\mathbb{X} \times[0, T]} \widehat{\phi}(x, t) \mu(d x d t)\right)
$$

satisfies

$$
\|\xi-\widehat{\xi}\|_{L_{2}(P)}=\min _{\theta \in \mathcal{H}}\|\xi-\theta\|_{L_{2}(P)} .
$$

\footnotetext{
${ }^{3}$ The stated convergence is a.s. and in $L_{1}$, but as the elements are in $L_{2}$ convergence in this sense follows.
} 
Naturally, $\widehat{w}=E\left[\frac{\xi}{R_{T}}\right]$. The problem (4.9) is then equivalent to

$$
\operatorname{var}(\xi-\widehat{\xi})=\min _{\substack{\theta \in \mathcal{H} \\ w=E\left[\xi / R_{T}\right]}} \operatorname{var}(\xi-\theta) .
$$

The minimal variance hedging problem can be formulated for a whole process $\xi_{t}, 0 \leq t \leq T$, with $\xi_{t} \in L_{2}(P)$. In this case the wish is to find the density of investments $\widehat{\phi}$ and the initial endowment $\widehat{w}$ such that

$$
d \widehat{\xi}_{t}=\widehat{\xi}_{t} r_{t} d t+R_{t} \int_{\mathbb{X}} \widehat{\phi}(x, t) \mu(d x d t), \quad \widehat{\xi}_{0}=\widehat{w}
$$

is satisfying

$$
\left\|\xi_{t}-\widehat{\xi}_{t}\right\|_{L_{2}(P)}=\min _{\theta}\left\|\xi_{t}-\theta_{t}\right\|_{L_{2}(P)}, \quad \forall t \in[0, T] .
$$

The minimum is taken over all processes $\theta_{t}, 0 \leq t \leq T$, representing the value process of some strategy, i.e. given by

$$
d \theta_{t}=\theta_{t} r_{t} d t+R_{t} \int_{\mathbb{X}} \varphi(x, t) \mu(d x d t), \quad \theta_{0}=w,
$$

for some $\varphi \in L_{2}(\mathfrak{P})$ and $w \in \mathbb{R}$.

Theorem 4.2. Any claim $\xi \in L_{2}(P)$ admits minimal variance hedge $\widehat{\xi}$ achievable via the minimal variance hedging strategy characterized by an initial endowment

$$
\widehat{w}=E\left[\frac{\xi}{R_{T}} \mid \mathcal{F}_{0}\right]
$$

and a density of investment

$$
\widehat{\phi}(x, t)=\mathfrak{D}_{x, t} \frac{\xi}{R_{T}}, \quad(x, t) \in \mathbb{X} \times[0, T] .
$$

Proof. This is a direct consequence of Theorem 3.1. We omit the details.

Theorem 4.3. Let $\xi_{t}, 0 \leq t \leq T$, be a given stochastic process of desired payoffs in the course of time. If its discount process

$$
\bar{\xi}_{t}:=\frac{\xi_{t}}{R_{t}}, \quad 0 \leq t \leq T,
$$

is a martingale w.r.t. $\mathbb{F}$ in $L_{2}(P)$, then the minimal variance hedge process

$$
\widehat{\xi}_{t}=R_{t}\left(\widehat{w}+\int_{\mathbb{X} \times[0, t]} \widehat{\phi}(x, s) \mu(d x d s)\right)
$$

is obtained with the strategy

$$
\begin{aligned}
& \widehat{w}=\xi_{0} \\
& \widehat{\phi}(x, s)=\mathfrak{D}_{x, s} \frac{\xi_{T}}{R_{T}}, \quad(x, s) \in \mathbb{X} \times[0, T] .
\end{aligned}
$$


Proof. For any $t$, by application of Theorem 3.1 we have

$$
\bar{\xi}_{t}=\bar{\xi}_{t}^{(0)} \oplus \int_{\mathbb{X} \in[0, t]} \mathfrak{D}_{x, s} \bar{\xi}_{t} \mu(d x d z) \quad\left(\mathfrak{D} \bar{\xi}_{t}^{(0)} \equiv 0\right) .
$$

Set

$$
\widehat{\bar{\xi}}_{t}:=E\left[\bar{\xi}_{t}\right]+\int_{\mathbb{X} \times[0, t]} \mathfrak{D}_{x, s} \bar{\xi}_{t} \mu(d x d s)
$$

and $\widehat{\xi}:=R_{t} \widehat{\bar{\xi}}_{t}$. Then

$$
\begin{aligned}
E\left[\left(\xi_{t}-\widehat{\xi}_{t}\right)^{2}\right] & =E\left[R_{t}^{2}\left(\bar{\xi}_{t}-\widehat{\bar{\xi}}_{t}\right)^{2}\right] \\
& \leq E\left[R_{t}^{2}\left(\bar{\xi}_{t}-w-\int_{\mathbb{X} \times[0, t]} \varphi(x, s) \mu(d x d s)\right)^{2}\right] \\
& =E\left[\left(\xi_{t}-\theta_{t}\right)^{2}\right],
\end{aligned}
$$

for all processes $\theta_{t}=R_{t}\left(w+\int_{\mathbb{X} \times[0, t]} \varphi(x, s) \mu(d x d s)\right)$. On the other side, being $\bar{\xi}_{t}, 0 \leq t \leq T$, a martingale, then

$$
\bar{\xi}_{t}=E\left[\bar{\xi}_{T} \mid \mathcal{F}_{t}\right]=E\left[\bar{\xi}_{T}^{(0)} \mid \mathcal{F}_{t}\right] \oplus \int_{\mathbb{X} \times[0, t]} \mathfrak{D}_{x, s} \bar{\xi}_{T} \mu(d x d s) .
$$

Thus $\mathfrak{D}_{x, s} \bar{\xi}_{T}=\mathfrak{D}_{x, s} \bar{\xi}_{t},(x, s) \in \mathbb{X} \times[0, T]$, and

$$
\widehat{\xi}_{t}=R_{t}\left(\xi_{0}+\int_{\mathbb{X} \times[0, T]} \mathfrak{D}_{x, s} \frac{\xi_{T}}{R_{T}} \mu(d x d s)\right) .
$$

Corollary 4.1. Let $\xi_{t}, 0 \leq t \leq T$, be a given stochastic process of desired payoffs in the course of time. If its discount process $\bar{\xi}_{t}, 0 \leq t \leq T$, is a martingale w.r.t. $\mathbb{F}$ only on some subset $\mathbb{T} \subseteq[0, T]$ with $T \in \mathbb{T}$, i.e. $E\left[\bar{\xi}_{t} \mid \mathcal{F}_{s}\right]=\bar{\xi}_{s}$, for $s, t \in \mathbb{T}$ such that $s \leq t$, then the process $\widehat{\xi}_{t}, 0 \leq t \leq T$, characterized in (4.11) is the minimal variance hedge in the sense that

$$
\left\|\xi_{t}-\widehat{\xi}_{t}\right\|_{L_{2}(P)}=\min _{\theta}\left\|\xi_{t}-\theta_{t}\right\|_{L_{2}(P)}, \quad \forall t \in \mathbb{T} .
$$

Cf. (4.10). Note that if $\mathbb{T}=\{T\}$, we recover the result of Theorem 4.2.

Corollary 4.2. If the the given process $\xi_{t}, 0 \leq t \leq T$, is such that $\xi_{T} \in \mathcal{H}$ and

$$
\frac{\xi_{t}}{R_{t}} \leq E\left[\frac{\xi_{T}}{R_{T}} \mid \mathcal{F}_{t}\right], \quad 0 \leq t \leq T .
$$

Then the process $\widehat{\xi}_{t}, 0 \leq t \leq T$, characterized in (4.11) is the minimal upper hedge in the sense that

$$
\xi_{t} \leq \widehat{\xi}_{t} \leq \widetilde{\xi}_{t}, \quad \forall t
$$

for any possible value $\widetilde{\xi}_{t}, 0 \leq t \leq T$, i.e.

$$
d \widetilde{\xi}_{t}=\widetilde{\xi}_{t} r_{t} d t+R_{t} \int_{\mathbb{X}} \varphi(x, t) \mu(d x d t), \quad \widetilde{\xi}_{0}=w
$$


The result can be put in relation with the so-called Snell envelope of $\xi_{t}$, $0 \leq t \leq T$. See e.g. [26].

Corollary 4.2 can be applied, for example, to the case

$$
\xi_{t}=\left(S_{t}(B)-K\right)^{+}, \quad 0 \leq t \leq T,
$$

if the call option $\xi_{T}=\left(S_{T}(B)-K\right)^{+}$written on the package $B$ with strike price $K$ is replicable on the market.

\subsection{Minimal variance hedging and partial information}

The information available on the market is modeled by the filtration $\mathbb{F}$. We call this full information. However, we can consider the case of a trader having less information at disposal during his decision making process. In this case it is reasonable to model this partial information with a sub-filtration $\mathbb{E}$ of $\mathbb{F}$ :

$$
\mathbb{E}=\left\{\mathcal{E}_{t}, 0 \leq t \leq T\right\} \quad \text { with } \quad \mathcal{E}_{t} \subseteq \mathcal{F}_{t}, \forall t .
$$

Accordingly, the trader's density of investment will be predictable with respect to the sub-filtration $\mathbb{E}$, i.e. measurable with respect to the $\sigma$-algebra $\mathfrak{P}_{\mathbb{E}}$ generated by

$$
F \times B \times(s, u], \quad F \in \mathcal{E}_{s}, B \in \mathfrak{B}, 0 \leq s<u \leq T
$$

- cf. (2.3). For any $\xi \in L_{2}(P)$, we can define the stochastic function $\mathfrak{D}_{\mathbb{E}} \xi$ as the limit

$$
\mathfrak{D}_{\mathbb{E}} \xi=\lim _{n \rightarrow \infty} \varphi_{n}, \quad \text { i.e. } \quad\|\mathfrak{D} \xi-\varphi\|_{L_{2}(P \times M)} \stackrel{n \rightarrow \infty}{\longrightarrow} 0,
$$

of the simple integrands

$$
\varphi_{n}(x, t):=\sum_{k=1}^{K_{n}} E\left[\xi \frac{\mu\left(\Delta_{n k}\right)}{E\left[M\left(\Delta_{n k}\right) \mid \mathcal{E}_{s_{n k}}\right]} \mid \mathcal{E}_{s_{n k}}\right] \chi_{\Delta_{n k}}(x, t), \quad(x, t) \in \mathbb{X} \times[0, T],
$$

where the sets $\Delta_{n k}$ are the elements of $\mathcal{A}_{n}$. Compare with the fomula (3.8) for the non-anticipating derivative $\mathfrak{D} \xi$. In this case a decomposition similar to (3.10) holds:

Theorem 4.4. Any element $\xi \in L_{2}(P)$ admits integral representation as

$$
\xi=\xi_{\mathbb{E}}^{(0)} \oplus \int_{\mathbb{X} \times[0, T]} \mathfrak{D}_{\mathbb{E}, x, t} \xi \mu(d x d t)
$$

where $\xi_{\mathbb{E}}^{(0)} \in L_{2}(P)$. 
Comparing (3.10) and (4.14) we see that $\left\|\xi^{(0)}\right\|_{L_{2}(P)} \leq\left\|\xi_{\mathbb{E}}^{(0)}\right\|_{L_{2}(P)}$.

The minimal variance hedge this partially informed trader can achieve is characterized as follows. This is the corresponding result to Theorem 4.2 in the partial information context.

Theorem 4.5. Let $\xi \in L_{2}(P)$ be a given payoff. The minimal variance hedge $\widehat{\xi_{\mathbb{E}}}$ :

$$
\widehat{\xi}_{\mathbb{E}}=R_{t} \widehat{w}_{\mathbb{E}}+R_{t} \int_{\mathbb{X} \times[0, T]} \widehat{\phi}_{\mathbb{E}} \mu(d x d t),
$$

that a partially informed trader can achieve relying only on the information $\mathbb{E}$ is given by the initial endowment

$$
\widehat{w}_{E} E=E\left[\frac{\xi}{R_{T}}\right]
$$

and the density of investments

$$
\widehat{\phi}_{\mathbb{E}}(x, t)=\mathfrak{D}_{\mathbb{E}, x, t} \frac{\xi}{R_{T}}, \quad(x, t) \in \mathbb{X} \times[0, T] .
$$

Similarly we can derive the corresponding result to Theorem 4.3.

\section{$5 \quad$ Examples of large markets}

\subsection{Large market with countable number of assets}

With the notation introduced, the risky securities are indexed by the discrete topological space $\mathbb{X}=\{1,2, \ldots\}$. To any $x \in \mathbb{X}$ we associate the $\mathbb{F}$-adapted price process

$$
S_{t}(x), \quad 0 \leq t \leq T .
$$

A riskless asset is also available with price process given by

$$
R_{t}, \quad 0 \leq t \leq T,
$$

cf. (4.2). As indicated (cf. Remark 4.1), we assume that the excessive return process associated with any $x \in \mathbb{X}, \eta(x)$ given by

$$
\eta_{t}(x):=\frac{S_{t}(x)}{R_{t}}-\frac{S_{0}(x)}{R_{0}}, \quad 0 \leq t \leq T,
$$

is a square integrable martingale, and that for any distinct $x, y \in \mathbb{X}$ the corresponding excessive return processes have conditionally non-correlated increments, i.e.

$$
E\left[\left(\eta_{u}(x)-\eta_{s}(x)\right)\left(\eta_{u}(y)-\eta_{s}(y)\right) \mid \mathcal{F}_{s}\right]=0, \quad s \leq u .
$$


The excessive returns generated by holding different collections of assets can now be represented by the martingale random field

$$
\mu(\Delta):=\sum_{x \in \mathbb{X}} \int_{0}^{T} \chi_{\Delta}(x, t) d \eta_{t}(x), \quad \Delta \in \mathfrak{B}_{\mathbb{X}} \times \mathfrak{B}_{[0, T]} .
$$

The conditional variance and variance measures are then represented as

$$
M(\Delta):=\sum_{x \in \mathbb{X}} \int_{0}^{T} \chi_{\Delta}(x, t) d M_{t}(x), \quad \Delta \in \mathfrak{B}_{\mathbb{X}} \times \mathfrak{B}_{[0, T]},
$$

where $M_{t}(x):=\langle\eta(x)\rangle_{t}, 0 \leq t \leq T$, and

$$
m(\Delta):=E\left[\mu^{2}(\Delta)\right]=E\left[\sum_{x \in \mathbb{X}} \int_{0}^{T} \chi_{\Delta}(x, t) d M_{t}(x)\right] .
$$

Remark 5.1. The orthogonality of the martingale random field ensures that $L_{2}(\mathfrak{P})$ coincides with the generalized integrands defined in [12, Definition 2]. This is not the case without orthogonality (cf. [12, Example 1]).

Remark 5.2. In the case the investments are restricted only to a finite number of assets, then it is well-known that, given the initial endowment $w$ and the density of investments $\phi$, the trading strategy can be made self financing by holding the number

$$
\int_{\mathbb{X} \times[0, t]} \phi(x, s) \mu(d x d s)+w-\frac{\sum_{x \in \mathbb{X}} \phi(x, t) S_{t}(x)}{R_{t}}
$$

of units of riskless asset at any time $t$. As pointed out in [8] this observation is not trivially extended to general densities of investments involving an infinite number of assets because the sum $\sum_{x \in \mathbb{X}} \phi(x, t) S_{t}(x)$ is not necessarily welldefined. So even if the process $\int_{\mathbb{X} \times[0, t]} \phi(x, s) \mu(d x d s), 0 \leq t \leq T$, exists as the excessive return process associated with a self-financing trading strategy, it may be impossible to specify either the part invested in the riskless or risky assets.

In the sequel we consider a model of large markets already studied in [23] and [5]. See also [8]. Assume that $W, W(1), W(2), \ldots$ are independent Brownian motions and that the asset prices are given by the dynamics

$$
d R_{t}=R_{t} r d t
$$

and

$$
d S_{t}(i)=S_{t}(i)\left(r d t+\sigma_{i} d W_{t}(i)+\beta_{i} d W_{t}\right), \quad i=1,2, \ldots
$$


where $r$ and the $\sigma_{i} \neq 0$ and the $\beta_{i} \neq 0$ are uniformly bounded constants. Assume that one may also invest in another risky asset with price dynamics

$$
d S_{t}=S_{t}\left(r d t+\sigma d W_{t}\right) \quad \sigma \neq 0,
$$

which we call the infinitely diversified portfolio as in [5]. Consider now the self-financing trading strategy $(\pi, \theta)$ where $\pi$ denotes the number of the $i$ th asset held and $\theta$ the number of the infinitely diversified porfolio held. The wealth dynamics associated with this strategy is

$$
d V_{t}=V_{t} r d t+\pi_{t} S_{t}(i) \sigma d W_{t}(i)+\left(\pi_{t} S_{t}(i) \beta_{i}+\theta_{t} S_{t} \sigma\right) d W_{t}, \quad 0 \leq t \leq T .
$$

Hence, if the portfolio is balanced such that

$$
\theta_{t}=-\pi_{t} \frac{\beta_{i} S_{t}(i)}{\sigma S(t)}, \quad 0 \leq t \leq T
$$

then

$$
d V_{t}=V_{t} r d t+\pi_{t} S_{t}(i) \sigma_{i} d W_{t}(i)=V_{t}\left(r d t+\hat{\sigma}_{i} d W_{t}(i)\right), \quad 0 \leq t \leq T
$$

where $\hat{\sigma}_{i}=\sigma_{i} /\left(1-\frac{\beta_{i}}{\sigma}\right)$.

We can model the risky assets using the space $\mathbb{X}:=\{0,1, \ldots\}$ where the price dynamics of the asset $x \in \mathbb{X}$ are given by

$$
d \hat{S}_{t}(0)=\hat{S}_{t}(0)\left(r d t+\sigma d W_{t}\right)
$$

and

$$
d \hat{S}_{t}(x)=\hat{S}_{t}(x)\left(r d t+\hat{\sigma}_{x} d W_{t}(x)\right), \quad x=1,2, \ldots
$$

Setting

$$
\mu(\Delta):=\sum_{x=1}^{\infty} \int_{0}^{T} \chi_{\Delta}(x, t) d \eta_{t}(x), \quad \Delta \in \mathfrak{B}_{\mathbb{X}} \times \mathfrak{B}_{[0, T]}
$$

where

$$
\eta_{t}(x):=\frac{\hat{S}_{t}(x)}{R_{t}}-\frac{\hat{S}_{0}(x)}{R_{0}}, \quad 0 \leq t \leq T
$$

we are in the situation described in Example 2.2 and consider the model within the martingale random fields approach of Section 4. 


\subsection{Large market with a continuum of assets}

Take $\mathbb{X}:=[0, T]$ and suppose that $\lambda$ is a $\sigma$-finite measure on $\mathfrak{B}_{\mathbb{X}}$. Suppose that for any $x \in[0, T]$ there is a defaultable zero-coupon bond with face value 1 . and maturity $x$ and let $P_{t}(x)$ denote the time $t$ price of the bond. Clearly $P_{x}(x)=1$, if the bond has not defaulted, and some $\mathcal{F}_{x}$-measurable recovery value in $[0,1]$ otherwise. We assume that the for any $t \leq x$ the discounted price $\frac{P_{t}(x)}{R_{t}} \in L_{2}(P)$ and satisfies

$$
\frac{P_{t}(x)}{R_{t}}=E\left[\frac{P_{u}(x)}{R_{u}} \mid \mathcal{F}_{t}\right], \quad t \leq u \leq x .
$$

For $t>x$ we take $P_{t}(x):=\frac{R_{t}}{R_{x}} P_{x}(x)$ so that for any $x \in \mathbb{X}$ the excessive return process $\eta(x)$ given by

$$
\eta_{t}(x):=\frac{P_{t}(x)}{R_{t}}-\frac{P_{0}(x)}{R_{0}}, \quad 0 \leq t \leq T,
$$

is a square integrable martingale w.r.t. $\mathbb{F}$. Moreover, we assume that the excessive return processes of bonds of distinct maturities $x, y \in \mathbb{X}$ have conditionally non-correlated increments, i.e.

$$
E\left[\left(\eta_{t}(x)-\eta_{s}(x)\right)\left(\eta_{t}(y)-\eta_{s}(y)\right)\right]=0 \quad s, t \in[0, T]
$$

Define

$$
\mu(\Delta):=\int_{\mathbb{X} \times[0, T]} \chi_{\Delta}(x, t) d \eta_{t}(x) \lambda(d x)
$$

and correspondingly

$$
M(\Delta)=\int_{\mathbb{X} \times[0, T]} \chi_{\Delta}(x, t) d M_{t}(x) d \lambda(x)
$$

and

$$
m(\Delta)=\int_{[0, T]} E\left[\int_{\mathbb{X}} \chi_{\Delta}(x, t) d M_{t}(x)\right] d \lambda(x)
$$

with $M(x)=<\eta(x)>$. The set-function $\mu$ has the properties of a martingale random field. In particular, suppose $m(\Delta)<\infty$ and let $I_{+}$denote the subset of $\mathbb{X}$ on which

$$
\int_{[0, T]} \chi_{\Delta}(x, t) d \eta_{t}(x)
$$

is non-negative and define $\Delta_{+}:=\Delta \cap I_{+} \times[0, T]$ and $\Delta_{-}:=\Delta \cap \Delta_{+}^{C}$. Clearly 
$\mu\left(\Delta_{-}\right) \leq 0 \leq \mu\left(\Delta_{+}\right)$, both being integrable. Let $\Delta \in \mathfrak{B}_{\mathbb{X}} \times \mathfrak{B}_{(t, T]}$

$$
\begin{aligned}
E\left[\mu(\Delta) \mid \mathcal{F}_{t}\right] & =E\left[\mu\left(\Delta_{+}\right)+\mu\left(\Delta_{-}\right) \mid \mathcal{F}_{t}\right] \\
& =E\left[\int_{[0, T]} \int_{[0, T]} \chi_{\Delta_{+}}(x, s) d \eta_{s}(x) \lambda(d x) \mid \mathcal{F}_{t}\right] \\
& +E\left[\int_{[0, T]} \int_{[0, T]} \chi_{\Delta_{-}}(x, s) d \eta_{s}(x) \lambda(d x) \mid \mathcal{F}_{t}\right] \\
& =\int_{[0, T]} E\left[\int_{[0, T]} \chi_{\Delta_{+}}(x, s) d \eta_{s}(x) \mid \mathcal{F}_{t}\right] \lambda(d x) \\
& +\int_{[0, T]} E\left[\int_{[0, T]} \chi_{\Delta_{-}}(x, s) d \eta_{s}(x) \mid \mathcal{F}_{t}\right] \lambda(d x) \\
& =\int_{[0, T]} E\left[\int_{[0, T]} \chi_{\Delta}(x, s) d \eta_{s}(x) \mid \mathcal{F}_{t}\right] \lambda(d x) \\
& =0,
\end{aligned}
$$

by the Tonelli theorem.

Proceeding in a similar manner and exploiting the conditional orthogonality of the price processes, we can prove that if $m\left(\Delta_{1}\right)$ and $m\left(\Delta_{2}\right)$ are finite and $\Delta_{1}$ and $\Delta_{2}$ are disjoint then

$$
E\left[\mu\left(\Delta_{1}\right) \mu\left(\Delta_{2}\right)\right]=0 .
$$

Hence $\mu$ is a martingale random field.

\section{References}

[1] F.E. Benth, G. Di Nunno, A. Løkka, B. Øksendal, and F. Proske. Explicit representation of the minimal variance portfolio in markets driven by Lévy processes. Preprint available at http://www.math.uio.no/ eprint/pure_math/, no 27/2001.

[2] T.F. Bewley. Existence of equilibria in economies with infinitely many commodities. J. Econom. Theory, 4:514-540, 1972.

[3] P. Billingsley. Probability and Measure. Wiley, 1995.

[4] T. Bjork, G Di Masi, Y. Kabanov, and W. Runggaldier. Towards a general theory of bond markets. Finance and Stochastics, 1:141-174, 1997.

[5] T. Björk and B. Näslund. Diversified portfolios in continuous time. European Finance Review, 1:361-387, 1998.

[6] R. Cairoli and J. Walsh. Stochastic integrals in the plane. Acta Math., 134:111-183, 1975. 
[7] L. Campi. Mean-variance hedging for large financial markets. To appear in Stochastic Analysis and Applications. Preprint version at http:// www.geocities.com/lucianocampi/.

[8] M. De Donno. A note on completeness in large financial markets. Mathematical Finance, 14:295-315, 2004.

[9] M. De Donno. The term structure of interest rates as a random field: A stochastic integration approach. In Stochastic Processes and Applications to Mathematical Finance, Proceedings of the Ritsumeikan International Symposium, pages 27-51, 2004.

[10] M. De Donno, P. Guasoni, and M. Pratelli. Super-replication and utility maximization in large financial markets. Stochastic Process. Appl., 115:2006-2022, 2005.

[11] M. De Donno and M. Pratelli. On the use of measure-valued processes in bond markets. Finance and Stochastics, 8:87-109, 2004.

[12] M. De Donno and M. Pratelli. Stochastic integration with respect to a sequence of semimartingales. In M. Émery and M. Yor, editors, Séminaire de Probabilités XXXIX, pages 119-135. Springer, 2006.

[13] G. Di Nunno. Random fields evolution: non-anticipating integration and differentiation. Theory of Probability and Math. Statistics, 66:8294, 2002.

[14] G. Di Nunno. Stochastic integral representations, stochastic derivatives and minimal variance hedging. Stochastics Stochastics Rep., 73:181$198,2002$.

[15] G. Di Nunno. Random fields: non-anticipating derivative and differentiation formulae. Infin. Dimens. Anal. Quantum Probab. Relat. Top., 10:465-481, 2007.

[16] G. Di Nunno, B. Øksendal, and F. Proske. White noise analysis for Lévy processes. Journal of Functional Analysis, 206:109-148, 2004.

[17] G. Di Nunno, B. Øksendal, and F. Proske. Malliavin Calculus For Lévy Processes with Applications to Finance. Springer, 2008.

[18] G. Di Nunno and Yu. A. Rozanov. On stochastic integration and differentiation. Acta Appl. Math., 58:231-235, 1999.

[19] G. Di Nunno and Yu. A. Rozanov. Stochastic integrals and adjoint derivatives. In Stochastic Analysis and Applications, volume 2. Springer, Berlin, 2007. Abel Symp.

[20] H. Föllmer and A. Schied. Stochastic Finance. Springer, 2002. 
[21] C. Gourieroux, J. P. Laurent, and H. Pham. Mean-variance hedging and numéraire. Mathematical Finance, 8:179-200, 1998.

[22] O. Hart. monopolistic competition in a large economy with differentiated commodities. Review of Economic Studies, 46:1-30, 1979.

[23] Y. Kabanov and D. O. Kramkov. Large financial markets: asymptotic arbitrage and contiguity. Probability Theory and its Applications, 39:222-229, 1994.

[24] Y. Kabanov and D. O. Kramkov. Asymptotic arbitrage in large financial markets. Finance and Stochastics, 2:143-172, 1998.

[25] O. Kallenberg. Foundations of Modern Probability. Springer, 1997.

[26] I. Karatzas and S.E. Shreve. Brownian Motion and Stochastic Calculus. Springer, 1998.

[27] D. P. Kennedy. The term structure of interest rate as a Gaussian random field. Mathematical Finance, 4:247-258, 1994.

[28] D. P. Kennedy. Characterizing Gaussian models of the term structure of interest rates. Mathematical Finance, 7:107-118, 1997.

[29] I. Klein. A fundamental theorem of asset prices for large financial markets. Theory of probability and its applications, 41:927-934, 1996.

[30] I. Klein and W. Schachermayer. Asymptotic arbitrage in non-complete large financial markets. Theory of probability and its applications, 41:927-934, 1996.

[31] I. Klein and W. Schachermayer. A quantitative and a dual version of the Halmos-Savage theorem with applications to mathematical finance. Annals of Probability, 24:867-861, 1996.

[32] O. Kreps. Arbitrage and equilibrium in economics with infinitely many commodities. Journal of Math. Econom., 8:15-35, 1981.

[33] J.A. Léon, J.L. Solé, F. Utzet, and J. Vives. On Lévy processes, Malliavin calculus and market models with jumps. Finance and Stochastics, 6:197-225, 2002.

[34] A. Løkka. Martingale representation and functionals of Lévy processes. Preprint 21/01, available at http://www. math.uio.no/eprint/, 2001.

[35] P. Malliavin. Stochastic Analysis. Springer-Verlag, New York, 1997.

[36] R. Mikulevicius and B.L. Rozovskii. Normalized stochastic integrals in topological vector spaces. In Séminaire de Probabilités XXXII, volume 1686. Springer, Berlin, 1998. 
[37] D. Nualart. The Malliavin Calculus and Related Topics. SpringerVerlag, New York, 1995.

[38] J. Picard. Formules de dualité sur l'espace de Poisson. Ann. IHP, Série Proba. et Stat., 32:509-548, 1996.

[39] P. Protter. Stochastic Integration and Differential Equations. Springer, 2005.

[40] M. M. Rao. Conditional measures and applications. Chapman and Hall, Boca Raton, FL, 2005.

[41] L.C.G. Rogers and D. Williams. Diffusions, Markov processes, and martingales, volume 2, Itô calculus, Reprint of the second (1994) edition. Cambridge University Press, Cambridge, 2000.

[42] K. Sato. Lévy Processes and Infinitely Divisible Distributions. Cambridge University Press, Cambridge, 1999.

[43] M. Schweizer. A guided tour through quadratic hedging approaches. In E. Jouini, J. Cvitanic, and M. Musiela, editors, Option Pricing, Interest Rates and Risk Management, pages 538-574. Cambridge University Press, 2001.

[44] E. Wong and M. Zakai. Martingales and stochastic integrals for processes with a multi-dimensional parameter. Z. Wahrscheinlichkeitstheorie und Verw. Gebiete, 29:109-122, 1974. 